\title{
On the mechanics of local-distortional interaction in thin-walled lipped channel beams
}

\author{
André D. Martins ${ }^{1}$, Dinar Camotim ${ }^{1}$, Rodrigo Gonçalves ${ }^{2}$, Pedro B. Dinis ${ }^{1}$
}

\begin{abstract}
This paper investigates the elastic post-buckling behavior of simply supported thin-walled lipped channel beams undergoing local-distortional (L-D) interaction. The beams are uniformly bent about the major-axis and experience flange-triggered local buckling (most common situation in practice). Three beams are analyzed, each exhibiting a different type of L-D interaction, namely (i) "true L-D interaction" (close local and distortional critical buckling moments), (ii 1 ) "secondary local-bifurcation interaction" and (ii 2 ) "secondary distortional-bifurcation interaction" (critical distortional/local buckling moments and high enough yield stresses). The results presented and discussed are obtained through geometrically non-linear Generalized Beam Theory (GBT) analyses and provide the evolution, along given equilibrium paths, of the beam deformed configuration (expressed in GBT modal form) and relevant displacement profiles, making it possible to acquire in-depth knowledge on the beam L-D interaction mechanics. Particular attention is devoted to interpreting the quantitative and qualitative differences exhibited by the beam post-buckling behaviors associated with the three aforementioned L-D interaction types.
\end{abstract}

\section{Introduction}

The complex thin-walled open cross-section shapes commonly exhibited by cold-formed steel members (e.g., with several intermediate and end stiffeners) makes them highly prone to several individual (local, distortional, global L, D, G) and/or coupled (L-D, L-G, D-G, L-D-G) buckling phenomena. Concerning the latter, establishing efficient design rules against interactive failures constitutes a considerable challenge ${ }^{3}$, much more so than in hot-rolled steel members, which are "only" affected by L-G interaction (which are already adequately handled by steel design specifications around the world). Traditionally, the non-linear behaviour of prismatic thin-walled members could only be rigorously assessed by resorting to time-consuming and computation-intensive shell finite element simulations, which provide outputs not easy to apprehend/interpret. However, in the last few years Generalized Beam Theory (GBT) has emerged as a very promising alternative to obtain similarly accurate results in a more efficient and (mostly) clarifying manner (only a few structurally meaningful d.o.f. are required). By performing GBTbased geometrically non-linear imperfect analyses (GNIA) of prismatic thin-walled members, it becomes possible to unveil and quantify the contributions of the various deformation modes to the member structural response under consideration. This feature makes it possible to acquire much deeper insight on that response, thus providing a much clearer picture of the mechanical aspects involved - i.e., GBT GNIA is an ideally suited numerical tool to investigate complex coupled instability problems, like the one addressed in this work.

${ }^{1}$ CERIS, ICIST, DECivil, Instituto Superior Técnico, Universidade de Lisboa, Portugal, <andrerdmartins@ist.utl.pt>, <dcamotim@civil.ist.pt>, <dinis@civil.ist.pt >.

2 CERIS, ICIST and Universidade Nova de Lisboa, Portugal, <rodrigo.goncalves@fct.unl.pt>.

3 Note that, as far as cold-formed steel columns are concerned, the authors proposed recently DSM-based strength curves that are able to handle L-D (Martins et al. 2016a) and L-D-G (Dinis et al. 2016, Dinis \& Camotim 2016) interactive failures. 
As far as thin-walled members affected by L-D interaction are concerned, GBT post-buckling results only exist for thin-walled lipped channel columns (Silvestre \& Camotim 2004, Martins et al. 2015a, 2016b) - to the authors' best knowledge, no such results are available for thin-walled beams. Therefore, the aim of this work is to present and discuss GBT numerical results concerning the geometrically non-linear behaviour of simply supported lipped channel beams under uniform major-axis bending and experiencing flange-triggered L-D interaction - these results shed fresh light on the mechanics underlying this coupling phenomenon, already unveiled by means of shell finite element analyses (Dinis \& Camotim 2010). It is worth noting that the beams analyzed are highly prone to L-D interaction due to the end supports (Martins et al. 2015b) - in fact, is very difficult to avoid this coupling phenomenon in the short-to-intermediate length range. Three beams are selected and analyzed, each of them exhibiting a different type of L-D interaction, namely (i) "true L-D interaction" (close local and distortional critical buckling moments), (ii) "secondary local-bifurcation interaction" and (iii) "secondary distortional-bifurcation interaction" (e.g., Martins et al. 2015b). The GBT elastic post-buckling results provide the evolution, along given equilibrium paths, of the beam deformed configuration (expressed in GBT modal form). Particular attention is paid to the characterization of the deformation patterns akin to the local and distortional critical buckling modes, and to relevant displacement longitudinal profiles, thus making it possible to acquire in-depth knowledge on the mechanics of beam L-D interaction. This knowledge will certainly impact the development of rational design rules for cold-formed steel beams failing in L-D interaction modes, which is currently under way (Martins et al. 2016c).

\section{GBT Geometrically Non-Linear Analysis}

The performance of a GBT structural analysis involves two (independent) main tasks, namely (i) a cross-section analysis, concerning the identification of the deformation modes and evaluation of the corresponding modal mechanical properties, and (ii) a member analysis (elastic buckling and post-buckling analyses, in the case of this paper), which consists of solving the appropriate differential equilibrium equation system. Next, a novel geometrically non-linear GBT formulation is presented and followed by a brief description of the most relevant deformations modes employed in the analysis of the lipped channel beams affected by L-D interaction.

\subsection{Formulation}

Consider the arbitrary open cross-section member depicted in Fig. 1, which also shows the reference system $x-s-z$ - coordinates along the member length, cross-section mid-line and wall thickness, respectively. As for $u(x, s)$, $v(x, s)$ and $w(x, s)$, they denote the corresponding displacement fields, also indicated in Fig. 1. According to GBT, the member mid-surface displacement field considers the variable separation

$$
u(x, s)=u_{k}(s) \phi_{k, x}(x) \quad v(x, s)=v_{k}(s) \phi_{k}(x) \quad w(x, s)=w_{k}(s) \phi_{k}(x)
$$

where (i) (.), $x=d(.) / d x$, (ii) Einstein's summation convention applies to subscript $k$, (iii) $\phi_{k}(x)$ are modal displacement amplitude functions defined along the member length (member analysis unknowns) and (iv) functions $u_{k}(s), v_{k}(s)$, $w_{k}(s)$ are the displacements profiles associated with deformation mode $k$, obtained from the cross-section analysis (briefly discussed in Section 2.3). The initial geometrical imperfections incorporated in the analyses are also expressed in GBT modal form - in this work, they are obtained from a preliminary GBT buckling analysis and subsequently normalized (as discussed in Section 4) - i.e., one has

$$
\bar{u}(x, s)=u_{k}(s) \bar{\phi}_{k, x}(x) \quad \bar{v}(x, s)=v_{k}(s) \bar{\phi}_{k}(x) \quad \bar{w}(x, s)=w_{k}(s) \bar{\phi}_{k}(x)
$$

GBT geometrically non-linear formulations were previously developed by Miosga (1976) and, much more recently by Silvestre (2005), Basaglia (2010) and Silva (2013). These authors adopted a total Lagrangian kinematic description and an additive decomposition of the strain terms into Green-Lagrange membrane strains and small-strain bending. The main novelty of the formulation developed in this paper is the consideration of the whole set of non-linear membrane strain terms, which is equivalent (but not similar) to the formulation recently proposed by Gonçalves \& Camotim (2012). The strain-displacement relationships adopted read 


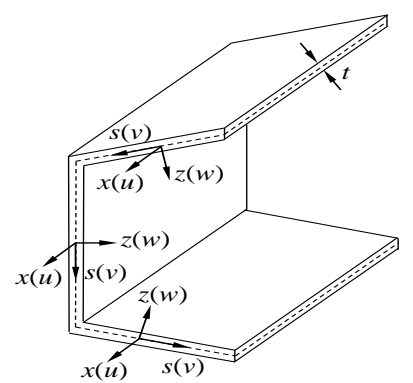

Figure 1: Arbitrary prismatic thin-walled member, local coordinate system and displacement components

$$
\begin{gathered}
\varepsilon_{x x}=u_{, x}-z w_{, x x}+\frac{1}{2}\left(u_{, x}^{2}+v_{, x}^{2}+w_{, x}^{2}\right)-\bar{u}_{, x}+z \bar{w}_{, x x}-\frac{1}{2}\left(\bar{u}_{, x}^{2}+\bar{v}_{, x}^{2}+\bar{w}_{, x}^{2}\right) \\
\varepsilon_{s s}=v_{, s}-z w_{, s s}+\frac{1}{2}\left(u_{, s}^{2}+v_{, s}^{2}+w_{, s}^{2}\right)-\bar{v}_{, s}+z \bar{w}_{, s s}-\frac{1}{2}\left(\bar{u}_{, s}^{2}+\bar{v}_{, s}^{2}+\bar{w}_{, s}^{2}\right) \\
\gamma_{x s}=u_{, s}+v_{, x}-2 z w_{, x s}+u_{, x} u_{, s}+v_{, x} v_{, s}+w_{, x} w_{, s}-\bar{u}_{, s}-\bar{v}_{, x}+2 z \bar{w}_{, x s}-\bar{u}_{, x} \bar{u}_{, s}-\bar{v}_{, x} \bar{v}_{, s}-\bar{w}_{, x} \bar{w}_{, s}
\end{gathered}
$$

The member strain energy is then expressed, in terms of the strain and stress components, as

$$
U=\frac{1}{2} \int_{V} \sigma_{i j} \varepsilon_{i j} d V=U_{1}+U_{2}+U_{3}-\left(\bar{U}_{1}+\bar{U}_{2}+\bar{U}_{3}\right)
$$

where $U_{i}$ and $\bar{U}_{i}(i=1,2,3)$ are the strain energy terms associated with the total deformation and initial geometric imperfections, respectively, given by

$$
\begin{aligned}
& U_{1}=\int_{L}\left(\frac{1}{2} C_{h i} \phi_{h, x x} \phi_{i, x x}+\frac{1}{2} B_{h i} \phi_{h} \phi_{i}+\frac{1}{2} D_{h i} \phi_{h, x} \phi_{i, x}+E_{h i} \phi_{h, x x} \phi_{i}\right) d x \\
& U_{2}=\int_{L}\left(\frac{1}{2} C_{h i j}^{I} \phi_{h, x x} \phi_{i, x} \phi_{j, x}+\frac{1}{2} B_{h i j}^{I} \phi_{h} \phi_{i} \phi_{j}+D_{h i j}^{I} \phi_{h, x} \phi_{i, x} \phi_{j}+\frac{1}{2} E_{h i j}^{I} \phi_{h, x x} \phi_{i} \phi_{j}+\frac{1}{2} E_{h i j}^{I I} \phi_{h} \phi_{i, x} \phi_{j, x}+\right. \\
& \left.+\frac{1}{2} C_{h i j}^{I I} \phi_{h, x x} \phi_{i, x x} \phi_{j, x x}+\frac{1}{2} B_{h i j}^{I I} \phi_{h} \phi_{i, x} \phi_{j, x}+D_{h i j}^{I I} \phi_{h, x} \phi_{i, x x} \phi_{j, x}+\frac{1}{2} E_{h i j}^{I I I} \phi_{h} \phi_{i, x x} \phi_{j, x x}+\frac{1}{2} E_{h i j}^{I V} \phi_{h, x} \phi_{i, x} \phi_{j, x x}\right) d x \\
& U_{3}=\int_{L}\left(\frac{1}{8} C_{h i j k}^{I} \phi_{h, x} \phi_{i, x} \phi_{j, x} \phi_{k, x}+\frac{1}{8} C_{h i j k}^{I I} \phi_{h, x x} \phi_{i, x x} \phi_{j, x x} \phi_{k, x x}+\frac{1}{4} C_{h i j k}^{I I I} \phi_{h, x x} \phi_{i, x x} \phi_{j, x} \phi_{k, x}+\frac{1}{8} B_{h i j k}^{I} \phi_{h} \phi_{i} \phi_{j} \phi_{k}+\frac{1}{8} B_{h i j k}^{I I} \phi_{h, x} \phi_{i, x} \phi_{j, x} \phi_{k, x}+\right. \\
& +\frac{1}{4} B_{h i j k}^{I I I} \phi_{h, x} \phi_{i, x} \phi_{j} \phi_{k}+\frac{1}{2} D_{h i j k}^{I} \phi_{h, x} \phi_{i} \phi_{j, x} \phi_{k}+\frac{1}{2} D_{h i j k}^{I I} \phi_{h, x x} \phi_{i, x} \phi_{j, x x} \phi_{k, x}+D_{h i j k}^{I I I} \phi_{h, x x} \phi_{i, x} \phi_{j, x} \phi_{k}+\frac{1}{4} E_{h i j k}^{I} \phi_{h, x} \phi_{i, x} \phi_{j} \phi_{k}+ \\
& \left.+\frac{1}{4} E_{h i j k}^{I I} \phi_{h, x} \phi_{i, x} \phi_{j, x x} \phi_{k, x x}+\frac{1}{4} E_{h i j k}^{I I I} \phi_{h, x} \phi_{i, x} \phi_{j, x} \phi_{k, x}+\frac{1}{4} E_{h i j k}^{I V} \phi_{h} \phi_{i} \phi_{j, x x} \phi_{k, x x}\right) d x \\
& \bar{U}_{1}=\int_{L}\left(C_{h i} \phi_{h, x x} \bar{\phi}_{i, x x}+B_{h i} \phi_{h} \bar{\phi}_{i}+D_{h i} \phi_{h, x} \bar{\phi}_{i, x}+E_{h i} \phi_{h, x x} \bar{\phi}_{i}+E_{i h} \phi_{h} \bar{\phi}_{i, x x}\right) d x \\
& \bar{U}_{2}=\int_{L}\left(\frac{1}{2} C_{h i j}^{I} \phi_{h, x x} \bar{\phi}_{i, x} \bar{\phi}_{j, x}+\frac{1}{2} B_{h i j}^{I} \phi_{h} \bar{\phi}_{i} \bar{\phi}_{j}+D_{h i j}^{I} \phi_{h, x} \bar{\phi}_{i, x} \bar{\phi}_{j}+\frac{1}{2} E_{h i j}^{I} \phi_{h, x x} \bar{\phi} \bar{\phi}_{j}+\frac{1}{2} E_{h i j}^{I I} \phi_{h} \bar{\phi}_{i, x} \bar{\phi}_{j, x}+\right. \\
& +\frac{1}{2} C_{h i j}^{I I} \phi_{h, x x} \bar{\phi}_{i, x x} \bar{\phi}_{j, x x}+\frac{1}{2} B_{h i j}^{I I} \phi_{h} \bar{\phi}_{i, x} \bar{\phi}_{j, x}+D_{h i j}^{I I} \phi_{h, x} \bar{\phi}_{i, x x} \bar{\phi}_{j, x}+\frac{1}{2} E_{h i j}^{I I I} \phi_{h} \bar{\phi}_{i, x x} \bar{\phi}_{j, x x}+\frac{1}{2} E_{i j h}^{I V} \phi_{h, x x} \bar{\phi}_{i, x} \bar{\phi}_{j, x}+ \\
& +\frac{1}{2} C_{j h i}^{I} \bar{\phi}_{j, x x} \phi_{h, x} \phi_{i, x}+\frac{1}{2} B_{j h i}^{I} \bar{\phi}_{j} \phi_{h} \phi_{i}+D_{j h i}^{I} \bar{\phi}_{j, x} \phi_{h, x} \phi_{i}+\frac{1}{2} E_{j h i}^{I} \bar{\phi}_{j, x x} \phi_{h} \phi_{i}+\frac{1}{2} E_{j h i}^{I I} \bar{\phi}_{j} \phi_{h, x} \phi_{i, x}+ \\
& \left.+\frac{1}{2} C_{h i j}^{I I} \bar{\phi}_{j, x x} \phi_{h, x x} \phi_{i, x x}+\frac{1}{2} B_{j h i}^{I I} \bar{\phi}_{j} \phi_{h, x} \phi_{i, x}+D_{j h i}^{I I} \bar{\phi}_{j, x} \phi_{h, x x} \phi_{i, x}+\frac{1}{2} E_{j h i}^{I I I} \bar{\phi}_{j} \phi_{h, x x} \phi_{i, x x}+\frac{1}{2} E_{h i j}^{I V} \bar{\phi}_{j, x x} \phi_{h, x} \phi_{i, x}\right) d x
\end{aligned}
$$

3 


$$
\begin{aligned}
\bar{U}_{3}= & \int_{L}\left(\frac{1}{4} C_{h i j k}^{I} \phi_{h, x} \phi_{i, x} \bar{\phi}_{j, x} \bar{\phi}_{k, x}+\frac{1}{4} C_{h k j i}^{I I} \phi_{h, x x} \phi_{i, x x} \bar{\phi}_{j, x x} \bar{\phi}_{k, x x}+\frac{1}{4} C_{h i j k}^{I I I} \phi_{h, x x} \phi_{i, x x} \bar{\phi}_{j, x} \bar{\phi}_{k, x}+\frac{1}{4} C_{j k h i}^{I I I} \phi_{h, x} \phi_{i, x} \bar{\phi}_{j, x x} \bar{\phi}_{k, x x}+\right. \\
& +\frac{1}{4} B_{h i j k}^{I} \phi_{h} \phi_{i} \bar{\phi}_{j} \bar{\phi}_{k}+\frac{1}{4} B_{h k j i}^{I I} \phi_{h, x} \phi_{i, x} \bar{\phi}_{j, x} \bar{\phi}_{k, x}+\frac{1}{4} B_{h i j k}^{I I I} \phi_{h, x} \phi_{i, x} \bar{\phi}_{j} \bar{\phi}_{k}+\frac{1}{4} B_{j k h i}^{I I} \phi_{h} \phi_{i} \bar{\phi}_{j, x} \bar{\phi}_{k, x}+D_{h i j k}^{I} \phi_{h, x} \phi_{i} \bar{\phi}_{j, x} \bar{\phi}_{k}+ \\
& +D_{h i j k}^{I I} \phi_{h, x x} \phi_{i, x} \bar{\phi}_{j, x x} \bar{\phi}_{k, x}+D_{h i j k}^{I I I} \phi_{h, x x} \phi_{i, x} \bar{\phi}_{j, x} \bar{\phi}_{k}+D_{j k h i}^{I I I} \phi_{h, x} \phi_{i} \bar{\phi}_{j, x x} \bar{\phi}_{k, x}+\frac{1}{4} E_{h i k}^{I} \phi_{h, x} \phi_{i, x} \bar{\phi}_{j} \bar{\phi}_{k}+\frac{1}{4} E_{j k h i}^{I} \phi_{h} \phi_{i} \bar{\phi}_{j, x} \bar{\phi}_{k, x}+ \\
& +\frac{1}{4} E_{h i j k}^{I I} \phi_{h, x} \phi_{i, x} \bar{\phi}_{j, x x} \bar{\phi}_{k, x x}+\frac{1}{4} E_{j k h i}^{I I} \phi_{h, x x} \phi_{i, x x} \bar{\phi}_{j, x} \bar{\phi}_{k, x}+\frac{1}{4} E_{h i j k}^{I I I} \phi_{h, x} \phi_{i, x} \bar{\phi}_{j, x} \bar{\phi}_{k, x}+\frac{1}{4} E_{j k h i}^{I I I} \phi_{h, x} \phi_{i, x} \bar{\phi}_{j, x} \bar{\phi}_{k, x}+ \\
& \left.+\frac{1}{4} E_{h i j k}^{I V} \phi_{h} \phi_{i} \bar{\phi}_{j, x x} \bar{\phi}_{k, x x}+\frac{1}{4} E_{j k h i}^{I V} \phi_{h, x x} \phi_{i, x x} \bar{\phi}_{j} \bar{\phi}_{k}\right) d x
\end{aligned}
$$

where (i) $C_{h i}, B_{h i}, D_{h i}, E_{h i}$ are second-order tensors whose components are modal mechanical properties characterizing the cross-section linear behavior, (ii) $C_{h i j}^{I}, C_{h i j}^{I I}, B_{h i j}^{I}, B_{h i j}^{I I}, D_{h i j}^{I}, D_{h i j}^{I I}, E_{h i j}^{I}, E_{h i j}^{I I}, E_{h i j}^{I I I}, E_{h i j}^{I V}$ are third-order tensors and (iii) $C_{h i j k}^{I}, C_{h i j k}^{I I}, C_{h i j k}^{I I I}, B_{h i j k}^{I}, B_{h i j k}^{I I}, B_{h i j k}^{I I I}, D_{h i j k}^{I I}, D_{h i j k}^{I I}, D_{h i j k}^{I I I}, E_{h i j k}^{I}, E_{h i j k}^{I I}, E_{h i j k}^{I I I}, E_{h i j k}^{I V}$ are fourth-order tensors, both associated with the member geometrically non-linear behavior - note that tensors $C, B, D$ tensors stem from the longitudinal extensions, transverse extensions and shear strains, while tensor $\boldsymbol{E}$ is associated with the coupling between longitudinal and transverse extensions (Poisson effects). These components, which are obtained exclusively on the basis of the cross-section deformations modes, are given by the expressions ${ }^{4}$

$$
\begin{array}{ll}
C_{h i}=Q_{11} \int_{A}\left(u_{h}-z w_{h}\right)\left(u_{i}-z w_{i}\right) d A & B_{h i}=Q_{22} \int_{A}\left(v_{h, s}-z w_{h, s s}\right)\left(v_{i, s}-z w_{i, s s}\right) d A \\
D_{h i}=Q_{66} \int_{A}\left(u_{h, s}+v_{h}-2 z w_{h, s}\right)\left(u_{i, s}+v_{i}-2 z w_{i, s}\right) d A & E_{h i}=Q_{12} \int_{A}\left(u_{h}-z w_{h}\right)\left(v_{i, s}-z w_{i, s s}\right) d A \\
C_{h i j}^{I}=Q_{11} \int_{A} u_{h}\left(v_{i} v_{j}+w_{i} w_{j}\right) d A & C_{h i j}^{I I}=Q_{11} \int_{A} u_{h} u_{i} u_{j} d A \\
B_{h i j}^{I}=Q_{22} \int_{A} v_{h, s}\left(v_{i, s} v_{j, s}+w_{i, s} w_{j, s}\right) d A & B_{h i j}^{I I}=Q_{22} \int_{A} v_{h, s} u_{i, s} u_{j, s} d A \\
D_{h i j}^{I}=Q_{66} \int_{A}\left(u_{h, s}+v_{h}\right)\left(v_{i} v_{j, s}+w_{i} w_{j, s}\right) d A & D_{h i j}^{I I}=Q_{66} \int_{A}\left(u_{h, s}+v_{h}\right) u_{i} u_{j, s} d A \\
E_{h i j}^{I}=Q_{12} \int_{A}^{A} u_{h}\left(v_{i, s} v_{j, s}+w_{i, s} w_{j, s}\right) d A & E_{h i j}^{I I}=Q_{12} \int_{A}^{\int} v_{h, s}\left(v_{i} v_{j}+w_{i} w_{j}\right) d A \\
E_{h i j}^{I I I}=Q_{12} \int_{A} v_{h, s} u_{i} u_{j} d A & E_{h i j}^{I V}=Q_{12} \int_{A} u_{h, s} u_{i, s} u_{j} d A \\
C_{h i j k}^{I}=Q_{11} \int_{A}\left(v_{h} v_{i}+w_{h} w_{i}\right)\left(v_{j} v_{k}+w_{j} w_{k}\right) d A & C_{h i j k}^{I I}=Q_{11} \int_{A} u_{h} u_{i} u_{j} u_{k} d A \\
B_{h i j k}^{I}=Q_{22} \int_{A}\left(v_{h, s} v_{i, s}+w_{h, s} w_{i, s}\right)\left(v_{j, s} v_{k, s}+w_{j, s} w_{k, s}\right) d A & C_{h i j k}^{I I I}=Q_{11} \int_{A} u_{h} u_{i}\left(v_{j} v_{k}+w_{j} w_{k}\right) d A \\
B_{h i j k}^{I I}=Q_{22} \int_{A} u_{h, s} u_{i, s} u_{j, s} u_{k, s} d A & B_{h i j k}^{I I I}=Q_{22} \int_{A} u_{h, s} u_{i, s}\left(v_{j, s} v_{k, s}+w_{j, s} w_{k, s}\right) d A \\
D_{h i j k}^{I}=Q_{66} \int_{A}\left(v_{h} v_{i, s}+w_{h} w_{i, s}\right)\left(v_{j} v_{k, s}+w_{j} w_{k, s}\right) d A & D_{h i j k}^{I I}=Q_{66} \int_{A} u_{h} u_{i, s} u_{j} u_{k, s} d A
\end{array}
$$

\footnotetext{
${ }^{4}$ Note that the determination of the fourth-order tensors can be (computationally) very costly if large numbers of deformation modes are included in the analysis - the order of magnitude of the computations is $p \times N M^{4}$, where $N M$ is the deformation mode number and $p$ is the number of the cross-section wall segments considered. In order to reduce drastically the time inherent to this procedure, measures were undertaken to simplify it, which account for (i) the deformation mode nature (e.g., if $h$ and/or $i$ and/or $j$ and/or $k$ is a shear mode, $C_{h i j k}^{\prime}$ is null) and (ii) symmetry properties (e.g., $C_{h i j k}^{\prime}$ is symmetric with respect to $(h, i)$ and $\left.(j, k)\right)$.
} 


$$
\begin{array}{ll}
D_{h i j k}^{I I I}=Q_{66} \int_{A} u_{h} u_{i, s}\left(v_{j} v_{k, s}+w_{j} w_{k, s}\right) d A & E_{h i j k}^{I I}=Q_{12} \int_{A} u_{h, s} u_{i, s} u_{j} u_{k} d A \\
E_{h i j k}^{I}=Q_{12} \int_{A}\left(v_{h} v_{i}+w_{h} w_{i}\right)\left(v_{j, s} v_{k, s}+w_{j, s} w_{k, s}\right) d A & E_{h i j k}^{I I I}=Q_{12} \int_{A} u_{h, s} u_{i, s}\left(v_{j} v_{k}+w_{j} w_{k}\right) d A \\
E_{h i j k}^{I V}=Q_{12} \int_{A}\left(v_{h, s} v_{i, s}+w_{h, s} w_{i, s}\right) u_{j} u_{k} d A &
\end{array}
$$

where $Q_{11}=Q_{22}=\frac{E}{1-v^{2}}, Q_{12}=v \frac{E}{1-v^{2}}, Q_{66}=\frac{E}{2(1+v)}$ ( $E$ and $v-$ Young's modulus and Poisson's ratio).

\subsection{Non-linear beam finite element and solution procedure}

The member analysis is performed by using (one-dimensional) beam finite elements similar to those developed by Silvestre \& Camotim 2003. The modal amplitude functions are approximated by means of linear combinations of (i) Lagrange cubic polynomial primitives $\psi_{i}^{L}(\xi), i=1, \ldots, 4$, for the deformations modes involving only warping displacements (axial extension and shear modes), and (i) Hermite cubic polynomials $\psi_{i}^{H}(\xi), i=1, \ldots, 4$, for the remaining conventional deformation modes and transverse extension modes - the various deformation mode families are addressed in Section 2.3. Such polynomials are the form

$$
\begin{array}{ll}
\psi_{1}^{H}=L_{e}\left(\xi^{3}-2 \xi^{2}+\xi\right) & \psi_{1, x}^{L}=\frac{1}{2}(1-\xi)(3 \xi-1)(3 \xi-2) \\
\psi_{2}^{H}=2 \xi^{3}-3 \xi^{2}+1 & \psi_{2, x}^{L}=\frac{9}{2} \xi(\xi-1)(3 \xi-2) \\
\psi_{3}^{H}=L_{e}\left(\xi^{3}-\xi^{2}\right) & \psi_{3, x}^{L}=\frac{9}{2} \xi(1-\xi)(3 \xi-1) \\
\psi_{4}^{H}=3 \xi^{2}-2 \xi^{3} & \psi_{4, x}^{L}=\frac{1}{2} \xi(3 \xi-1)(3 \xi-2)
\end{array}
$$

where $L_{e}$ is the finite element length and $\xi=x / L_{e}$. Therefore, the amplitude functions $\phi_{k}(x)$ associated with the conventional (except axial extension) and transverse extension modes are approximated through

$$
\phi_{k}(x)=\psi_{1}^{H} d_{k 1}+\psi_{2}^{H} d_{k 2}+\psi_{3}^{H} d_{k 3}+\psi_{4}^{H} d_{k 4}
$$

where $d_{k 1}=\phi_{k, x}(0), d_{k 2}=\phi_{k}(0), d_{k 3}=\phi_{k, x}\left(L_{e}\right), d_{k 4}=\phi_{k}\left(L_{e}\right)$ and the amplitude functions $\phi_{j, x}(x)$ associated with the deformation modes involving only warping are given by

$$
\phi_{j, x}(x)=\psi_{1, x}^{L} d_{j 1}+\psi_{2, x}^{L} d_{j 2}+\psi_{3, x}^{L} d_{j 3}+\psi_{4, x}^{L} d_{j 4}
$$

where $d_{k 1}=\phi_{k, x}(0), d_{k 2}=\phi_{k, x}\left(L_{e} / 3\right), d_{k 3}=\phi_{k, x}\left(2 L_{e} / 3\right), d_{k 4}=\phi_{k, x}\left(L_{e}\right)$

The finite element internal force vector is obtained by differentiating the strain energy equations (5.1)-(5.6), after the introduction of equations (10.1)-(10.2), to obtain

$$
f_{h \alpha}^{e}=f_{h \alpha}^{1}+f_{h \alpha}^{2}+f_{h \alpha}^{3}-\left(\bar{f}_{h \alpha}^{1}+\bar{f}_{h \alpha}^{2}+\bar{f}_{h \alpha}^{3}\right)
$$

where

$$
\begin{gathered}
f_{h \alpha}^{1}=\left(C_{h i} k_{\alpha \beta}^{22}+B_{h i} k_{\alpha \beta}^{00}+D_{h i} k_{\alpha \beta}^{11}+E_{h i} k_{\alpha \beta}^{20}+E_{i h} k_{\beta \alpha}^{20}\right) d_{i \beta} \\
f_{h \alpha}^{2}=\left(\frac{1}{2} C_{h i j}^{I} k_{\alpha \beta \eta}^{211}+C_{i h j}^{I} k_{\beta \alpha \eta}^{211}+\frac{1}{2} B_{h i j}^{I} k_{\alpha \beta \eta}^{000}+B_{i h j}^{I} k_{\beta \alpha \eta}^{000}+D_{h i j}^{I} k_{\alpha \beta \eta}^{110}+D_{i h j}^{I} k_{\beta \alpha \eta}^{110}+D_{j i h}^{I} k_{\eta \beta \alpha}^{110}+\right. \\
+\frac{1}{2} E_{h i j}^{I} k_{\alpha \beta \eta}^{200}+E_{i h j}^{I} k_{\beta \alpha \eta}^{200}+\frac{1}{2}\left(E_{h i j}^{I I}+B_{h i j}^{I I}\right) k_{\alpha \beta \eta}^{011}+\left(E_{i h j}^{I I}+B_{i h j}^{I I}\right) k_{\beta \alpha \eta}^{011}+\frac{3}{2} C_{h i j}^{I I} k_{\alpha \beta \eta}^{222}+ \\
\left.+D_{h i j}^{I I} k_{\alpha \beta \eta}^{121}+D_{i h j}^{I I} k_{\beta \alpha \eta}^{121}+D_{j i h}^{I I} k_{\eta \beta \alpha}^{121}+\frac{1}{2} E_{h i j}^{I I} k_{\alpha \beta \eta}^{022}+E_{i h j}^{I I} k_{\beta \alpha \eta}^{022}+E_{h i j}^{I V} k_{\alpha \beta \eta}^{112}+\frac{1}{2} E_{j i h}^{I V} k_{\eta \beta \alpha}^{122}\right) d_{i \beta} d_{j \eta}
\end{gathered}
$$




$$
\begin{aligned}
& f_{h \alpha}^{3}=\left(\frac{1}{2}\left(C_{h i j k}^{I}+B_{h i j k}^{I I}+E_{h i j k}^{I I I}\right) k_{\alpha \beta \eta \mu}^{1111}+\frac{1}{2} B_{h i j k}^{I} k_{\alpha \beta \eta \mu}^{0000}+\frac{1}{2}\left(E_{h i j k}^{I}+B_{h i j k}^{I I I}\right) k_{\alpha \beta \eta \mu}^{1100}+\frac{1}{2}\left(E_{j k h i}^{I}+B_{j k h i}^{I I I}\right) k_{\eta \mu \alpha \beta}^{1100}+\right. \\
& +\frac{1}{2}\left(D_{h i j k}^{I} k_{\alpha \beta \eta \mu}^{1010}+D_{i h j k}^{I} k_{\beta \alpha \eta \mu}^{1010}+D_{j i h k}^{I} k_{\eta \beta \alpha \mu}^{1010}+D_{k i j h}^{I} k_{\mu \beta \eta \alpha}^{1010}\right)+\frac{1}{2} C_{h i j k}^{I I} k_{\alpha \beta \eta \mu}^{2222}+\frac{1}{2} C_{h i j k}^{I I I} k_{\alpha \beta \eta \mu}^{2211}+\frac{1}{2} C_{j k k i}^{I I I} k_{\eta \mu \alpha \beta}^{2211}+ \\
& +D_{h i j k}^{I I} k_{\alpha \beta \eta \mu}^{2121}+\frac{1}{2} D_{i h j k}^{I I} k_{\beta \alpha \eta \mu}^{2121}+\frac{1}{2} D_{k i j h}^{I I} k_{\mu \beta \eta \eta \alpha}^{2121}+D_{h i j k}^{I I I} k_{\alpha \beta \eta \mu}^{2110}+D_{i h j k}^{I I I} k_{\beta \alpha \eta \mu}^{2110}+D_{j i h k}^{I I I} k_{\eta \beta \alpha \mu}^{2110}+D_{k i j h}^{I I I} k_{\mu \beta \eta \alpha}^{2110}+ \\
& \left.\frac{1}{2} E_{h i j k}^{I I} k_{\alpha \beta \eta \mu}^{I 122}+\frac{1}{2} E_{j k h k}^{I I} k_{\eta \mu \alpha \beta}^{1122}+\frac{1}{2} E_{j k h i}^{I I I} k_{\eta \mu \alpha \beta}^{1111}+\frac{1}{2} E_{h i j k}^{I V} k_{\alpha \beta \eta \mu}^{0022}+\frac{1}{2} E_{j k h i}^{I V} k_{\eta \mu \alpha \beta}^{0022}\right) d_{i \beta} d_{j \eta} d_{k \mu} \\
& \bar{f}_{h \alpha}^{1}=\left(C_{h i} k_{\alpha \beta}^{22}+B_{h i} k_{\alpha \beta}^{00}+D_{h i} k_{\alpha \beta}^{11}+E_{h i} k_{\alpha \beta}^{20}+E_{i h} k_{\beta \alpha}^{20}\right) \bar{d}_{i \beta} \\
& \bar{f}_{h \alpha}^{2}=\left(\frac{1}{2} C_{h i j}^{I} k_{\alpha \beta \eta}^{211}+\frac{1}{2} B_{h i j}^{I} k_{\alpha \beta \eta}^{000}+D_{h i j}^{I} k_{\alpha \beta \eta}^{110}+\frac{1}{2} E_{h i j}^{I} k_{\alpha \beta \eta}^{200}+\frac{1}{2}\left(E_{h i j}^{I I}+B_{h i j}^{I I}\right) k_{\alpha \beta \eta}^{011}+\frac{1}{2} C_{h i j}^{I I} k_{\alpha \beta \eta}^{222}+D_{h i j}^{I I} k_{\alpha \beta \eta}^{121}+\right. \\
& \left.+\frac{1}{2} E_{h i j}^{I I I} k_{\alpha \beta \eta}^{022}+\frac{1}{2} E_{i j h}^{I V} k_{\beta \eta \alpha}^{112}\right) \bar{d}_{i \beta} \bar{d}_{j \eta}+\left(C_{j h i}^{I} k_{\eta \alpha \beta}^{211}+B_{j h i}^{I} k_{\eta \alpha \beta}^{000}+D_{j h k}^{I} k_{\eta \alpha \beta}^{110}+D_{j i h}^{I} k_{\eta \beta \alpha}^{110}+E_{j h i}^{I} k_{\eta \alpha \beta}^{200}+\right. \\
& \left.+\left(E_{j h i}^{I I}+B_{j h i}^{I I}\right) k_{\eta \alpha \beta}^{011}+C_{h i j}^{I I} k_{\alpha \beta \eta}^{222}+D_{j h i}^{I I} k_{\eta \alpha \beta}^{121}+D_{j h}^{I I} k_{\eta \beta \alpha}^{121}+E_{j h i}^{I I I} k_{\eta \alpha \beta}^{022}+E_{h i j}^{I V} k_{\alpha \beta \eta}^{112}\right) d_{i \beta} \bar{d}_{j \eta} \\
& \bar{f}_{h \alpha}^{3}=\left(\frac{1}{2}\left(C_{h i j k}^{I}+E_{h i j k}^{I I I}\right) k_{\alpha \beta \eta \mu}^{1111}+\frac{1}{2} C_{h k j i}^{I I} k_{\alpha \mu \eta \beta}^{2222}+\frac{1}{2} C_{h i j k}^{I I I} k_{\alpha \beta \eta \mu}^{2211}+\frac{1}{2} C_{j k h i}^{I I I} k_{\eta \mu \alpha \beta}^{2211}+\frac{1}{2} B_{h i j k}^{I} k_{\alpha \beta \eta \mu}^{0000}+\frac{1}{2} B_{h k j i}^{I I} k_{\alpha \mu \eta \beta}^{1111}+\right. \\
& +\frac{1}{2}\left(E_{h i j k}^{I}+B_{h i j k}^{I I I}\right) k_{\alpha \beta \eta \mu}^{1100}+\frac{1}{2}\left(E_{j k h i}^{I}+B_{j k h i}^{I I I}\right) k_{\eta \mu \alpha \beta}^{1100}+D_{h i j k}^{I} k_{\alpha \beta \eta \mu}^{1010}+D_{i h j k}^{I} k_{\beta \alpha \eta \mu}^{1010}+D_{h i j k}^{I I} k_{\alpha \beta \eta \mu}^{2121}+D_{i h j k}^{I I} k_{\beta \alpha \eta \mu}^{2121}+ \\
& +D_{h i j k}^{I I I} k_{\alpha \beta \eta \mu}^{2110}+D_{i h j k}^{I I I} k_{\beta \alpha \eta \mu}^{2110}+D_{j k h i}^{I I I} k_{\eta \mu \alpha \beta}^{2110}+D_{j k i h}^{I I I} k_{\eta \mu \beta \alpha}^{2110}+\frac{1}{2} E_{h i k k}^{I I} k_{\alpha \beta \eta \mu}^{1122}+\frac{1}{2} E_{j k h i}^{I I} k_{\eta \mu \alpha \beta}^{1122}+\frac{1}{2} E_{j k h i}^{I I I} k_{\eta \mu \alpha \beta}^{1111}+ \\
& \left.+\frac{1}{2} E_{h i j k}^{I V} k_{\alpha \beta \eta \mu}^{0022}+\frac{1}{2} E_{j k h i}^{I V} k_{\eta \mu \alpha \beta}^{0022}\right) d_{i \beta} \bar{d}_{j \eta} \bar{d}_{k \mu}
\end{aligned}
$$

where (i) subscripts $h, i, j, k$ correspond to deformations modes associated with shape functions subscripts $\alpha, \beta, \eta, \mu$, respectively, and (ii) $k_{\alpha \beta}^{a b}, k_{\alpha \beta \eta}^{a b c}$ and $k_{\alpha \beta \eta \mu}^{a b c d}$ are defined by

$$
k_{\alpha \beta}^{a b}=\int_{L e} \frac{\partial^{a} \psi_{\alpha}}{\partial x^{a}} \frac{\partial^{b} \psi_{\beta}}{\partial x^{b}} d x \quad k_{\alpha \beta \eta}^{a b c}=\int_{L e} \frac{\partial^{a} \psi_{\alpha}}{\partial x^{a}} \frac{\partial^{b} \psi_{\beta}}{\partial x^{b}} \frac{\partial^{c} \psi_{\eta}}{\partial x^{c}} d x \quad k_{\alpha \beta \eta \mu}^{a b c d}=\int_{L e} \frac{\partial^{a} \psi_{\alpha}}{\partial x^{a}} \frac{\partial^{b} \psi_{\beta}}{\partial x^{b}} \frac{\partial^{c} \psi_{\eta}}{\partial x^{c}} \frac{\partial^{d} \psi_{\mu}}{\partial x^{d}} d x
$$

In order to perform/implement an incremental-iterative technique it is essential to evaluate the tangent stiffness matrix corresponding to for a given deformed configuration, obtained by differentiating the internal force vector $f_{h \alpha}^{e}$ (equation (11.1)) with respect to a given d.o.f $d_{i \beta}$ - one has

$$
T_{h i \alpha \beta}^{e}=T_{h i \alpha \beta}^{1}+T_{h i \alpha \beta}^{2}+T_{h i \alpha \beta}^{3}-\left(\bar{T}_{h i \alpha \beta}^{2}+\bar{T}_{h i \alpha \beta}^{3}\right)
$$

where

$$
\begin{gathered}
T_{h i \alpha \beta}^{1}=C_{h i} k_{\alpha \beta}^{22}+B_{h i} k_{\alpha \beta}^{00}+D_{h i} k_{\alpha \beta}^{11}+E_{h i} k_{\alpha \beta}^{20}+E_{i h} k_{\beta \alpha}^{20} \\
T_{h i \alpha \beta}^{2}=\left(C_{h i j}^{I} k_{\alpha \beta \eta}^{211}+C_{i h j}^{I} k_{\beta \alpha \eta}^{211}+C_{j h i}^{I} k_{\eta \alpha \beta}^{211}+B_{h i j}^{I} k_{\alpha \beta \eta}^{000}+B_{i h j}^{I} k_{\beta \alpha \eta}^{000}+B_{j h i}^{I} k_{\eta \alpha \beta}^{000}+D_{h i j}^{I} k_{\alpha \beta \eta}^{110}+\right. \\
+D_{h j i}^{I} k_{\alpha \eta \beta}^{110}+D_{i h j}^{I} k_{\beta \alpha \eta}^{110}+D_{j h k}^{I} k_{\eta \alpha \beta}^{110}+D_{j i h}^{I} k_{\eta \beta \alpha}^{110}+D_{i j h}^{I} k_{\beta \eta \alpha}^{110}+E_{h i j}^{I} k_{\alpha \beta \eta}^{200}+E_{i h j}^{I} k_{\beta \alpha \eta}^{200}+ \\
+E_{j h i}^{I} k_{\eta \alpha \beta}^{200}+\left(E_{h i j}^{I I}+B_{h i j}^{I I}\right) k_{\alpha \beta \eta}^{111}+\left(E_{i h j}^{I I}+B_{i h j}^{I I}\right) k_{\beta \alpha \eta}^{011}+\left(E_{j h i}^{I I}+B_{j h i}^{I I}\right) k_{\eta \alpha \beta}^{011}+3 C_{h i j}^{I I} k_{\alpha \beta \eta}^{222}+ \\
+D_{h i j}^{I I} k_{\alpha \beta \eta}^{121}+D_{h j i}^{I I} k_{\alpha \eta \beta}^{121}+D_{i h j}^{I l} k_{\beta \alpha \eta}^{121}+D_{j h i}^{I I} k_{\eta \alpha \beta}^{121}+D_{j i h}^{I I} k_{\eta \beta \alpha}^{121}+D_{i j h}^{I I} k_{\beta \eta \alpha}^{121}+E_{h i j}^{I I I} k_{\alpha \beta \eta}^{022}+ \\
\left.+E_{i h j}^{I I} k_{\beta \alpha \eta}^{222}+E_{j h i}^{I I} k_{\eta \alpha \beta}^{022}+E_{h i j}^{I V} k_{\alpha \beta \eta}^{112}+E_{h j i}^{I V} k_{\alpha \eta \beta}^{112}+E_{j i h}^{I I} k_{\eta \beta \alpha}^{112}\right) d_{j \eta}
\end{gathered}
$$




$$
\begin{aligned}
& T_{h i \alpha \beta}^{3}=\left(\frac{1}{2} C_{h i j k}^{I} k_{\alpha \beta \eta \mu}^{1111}+C_{h j i k}^{I} k_{\alpha \eta \beta \mu}^{1111}+\frac{1}{2} B_{h i j k}^{I} k_{\alpha \beta \eta \mu}^{0000}+B_{h j i k}^{I} k_{\alpha \eta \beta \mu}^{0000}+\frac{1}{2}\left(D_{h i j k}^{I} k_{\alpha \beta \eta \mu}^{1010}+D_{h j i k}^{I} k_{\alpha \eta \beta \mu}^{1010}+D_{h k j i}^{I} k_{\alpha \mu \eta \beta}^{1010}+\right.\right. \\
& +D_{i h j k}^{I} k_{\beta \alpha \eta \mu}^{1010}+D_{j h i k}^{I} k_{\eta \alpha \beta \mu}^{1010}+D_{k h j i}^{I} k_{\mu \alpha \eta \beta}^{1010}+D_{j i h k}^{I} k_{\eta \beta \alpha \mu}^{1010}+D_{i j h k}^{I} k_{\beta \eta \alpha \mu}^{1010}+D_{j k h i}^{I} k_{\eta \mu \alpha \beta}^{1010}+D_{k i j h}^{I} k_{\mu \beta \eta \alpha}^{1010}+D_{k j i h}^{I} k_{\mu \eta \beta \alpha}^{1010}+ \\
& \left.+D_{i k j h}^{I} k_{\beta \mu \eta \alpha}^{1010}\right)+\frac{1}{2} E_{h i j k}^{I} k_{\alpha \beta \eta \mu}^{1100}+E_{h j i k}^{I} k_{\alpha \eta \beta \mu}^{1100}+\frac{1}{2} E_{j k h i}^{I} k_{\eta \mu \alpha \beta}^{1100}+E_{i k h j}^{I} k_{\beta \mu \alpha \eta}^{1100}+\frac{3}{2} C_{h i j k}^{I I} k_{\alpha \beta \eta \mu}^{2222}+\frac{1}{2} C_{h i j k}^{I I I} k_{\alpha \beta \eta \mu}^{2211}+C_{h j i k}^{I I I} k_{\alpha \eta \beta \mu}^{2211}+ \\
& +\frac{1}{2} C_{j k h i}^{I I I} k_{\eta \mu \alpha \beta}^{2211}+C_{i k h j}^{I I I} k_{\beta \mu \alpha \eta}^{2211}+\frac{1}{2} B_{h i j k}^{I I I} k_{\alpha \beta \eta \mu}^{1100}+B_{h j i k}^{I I I} k_{\alpha \eta \beta \mu}^{1100}+\frac{1}{2} B_{j k h i}^{I I I} k_{\eta \mu \alpha \beta}^{1100}+B_{i k h j}^{I I I} k_{\beta \mu \alpha \eta}^{1100}+\frac{3}{2} B_{h i j k}^{I I} k_{\alpha \beta \eta \mu}^{1111}+2 D_{h i j k}^{I I} k_{\alpha \beta \eta \mu}^{2121}+ \\
& +D_{h j k}^{I I} k_{\alpha \eta \beta \mu}^{2121}+D_{i h j k}^{I I} k_{\beta \alpha \eta \mu}^{2121}+\frac{1}{2} D_{k h j i}^{I I} k_{\mu \alpha \eta \beta}^{2121}+\frac{1}{2} D_{k i j h}^{I I} k_{\mu \beta \eta \alpha}^{2121}+D_{k j i h}^{I I} k_{\mu \eta \beta \alpha}^{2121}+D_{h i j k}^{I I I} k_{\alpha \beta \eta \mu}^{2110}+D_{h j i k}^{I I I} k_{\alpha \eta \beta \mu}^{2110}+D_{h k j i}^{I I I} k_{\alpha \mu \eta \beta}^{2110}+D_{i h j k}^{I I I} k_{\beta \alpha \eta \mu}^{2110}+ \\
& +D_{j h i k}^{I I I} k_{\eta \alpha \beta \mu}^{2110}+D_{k h j i}^{I I I} k_{\mu \alpha \eta \beta}^{2110}+D_{j i h k}^{I I I} k_{\eta \beta \alpha \mu}^{2110}+D_{i j h k}^{I I I} k_{\beta \eta \alpha \mu}^{2110}+D_{j k h i}^{I I I} k_{\eta \mu \alpha \beta}^{2110}+D_{k i h h}^{I I I} k_{\mu \beta \eta \alpha}^{2110}+D_{k j i h}^{I I I} k_{\mu \eta \beta \alpha}^{2110}+D_{i k j h}^{I I I} k_{\beta \mu \eta \alpha}^{2110}+\frac{1}{2} E_{h i j k}^{I I} k_{\alpha \beta \eta \mu}^{1122}+ \\
& +E_{h j i k}^{I I} k_{\alpha \eta \beta \mu}^{1122}+\frac{1}{2} E_{j k h i}^{I I} k_{\eta \mu \alpha \beta}^{1122}+E_{i k h j}^{I I} k_{\beta \mu \alpha \eta}^{1122}+\frac{1}{2} E_{h i j k}^{I I I} k_{\alpha \beta \eta \mu}^{1111}+E_{h j i k}^{I I I} k_{\alpha \eta \beta \mu}^{1111}+\frac{1}{2} E_{j k h i}^{I I I} k_{\eta \mu \alpha \beta}^{1111}+E_{i k h j}^{I I I} k_{\beta \mu \alpha \eta}^{1111}+\frac{1}{2} E_{h i j k}^{I V} k_{\alpha \beta \eta \mu}^{0022}+E_{h j i k}^{I V} k_{\alpha \eta \beta \mu}^{0022}+ \\
& \left.+\frac{1}{2} E_{j k h i}^{I V} k_{\eta \mu \alpha \beta}^{0022}+E_{i k h j}^{I V} k_{\beta \mu \alpha \eta}^{0022}\right) d_{j \eta} d_{k \mu} \\
& \bar{T}_{h i \alpha \beta}^{2}=\left(C_{j h i}^{I} k_{\eta \alpha \beta}^{211}+B_{j h i}^{I} k_{\eta \alpha \beta}^{000}+D_{j h i}^{I} k_{\eta \alpha \beta}^{110}+D_{j i h}^{I} k_{\eta \beta \alpha}^{110}+E_{j h i}^{I} k_{\eta \alpha \beta}^{200}+\left(E_{j h i}^{I I}+B_{j h i}^{I I}\right) k_{\eta \alpha \beta}^{011}+C_{h i j}^{I I} k_{\alpha \beta \eta}^{222}+\right. \\
& \left.+D_{j h i}^{I I} k_{\eta \alpha \beta}^{121}+D_{j i h}^{I I} k_{\eta \beta \alpha}^{121}+E_{j h i}^{I I I} k_{\eta \alpha \beta}^{022}+E_{h i j}^{I V} k_{\alpha \beta \eta}^{112}\right) \bar{d}_{j \eta} \\
& \bar{T}_{h i \alpha \beta}^{3}=\left(\frac{1}{2}\left(C_{h i j k}^{I I}+E_{h i j k}^{I I I}\right) k_{\alpha \beta \eta \mu}^{1111}+\frac{1}{2} C_{h k j i}^{I I} k_{\alpha \mu \eta \beta}^{2222}+\frac{1}{2} C_{h i j k}^{I I I} k_{\alpha \beta \eta \mu}^{2211}+\frac{1}{2} C_{j k h i}^{I I I} k_{\eta \mu \alpha \beta}^{2211}+\frac{1}{2} B_{h i j k}^{I} k_{\alpha \beta \eta \mu}^{0000}+\frac{1}{2} B_{h k j i}^{I I} k_{\alpha \mu \eta \beta}^{0000}+\right. \\
& +\frac{1}{2}\left(E_{h i j k}^{I}+B_{h i j k}^{I I I}\right) k_{\alpha \beta \eta \mu}^{1100}+\frac{1}{2}\left(E_{j k h i}^{I}+B_{j k h i}^{I I I}\right) k_{\eta \mu \alpha \beta}^{1100}+D_{h i j k}^{I} k_{\alpha \beta \eta \mu}^{1010}+D_{i h j k}^{I} k_{\beta \alpha \eta \mu}^{1010}+D_{h i j k}^{I I} k_{\alpha \beta \eta \mu}^{2121}+D_{i h j k}^{I I} k_{\beta \alpha \eta \mu}^{2121}+ \\
& +D_{h i j k}^{I I I} k_{\alpha \beta \eta \mu}^{2110}+D_{i h j k}^{I I I} k_{\beta \alpha \eta \mu}^{2110}+D_{j k h i}^{I I I} k_{\eta \mu \alpha \beta}^{2110}+D_{j k i h}^{I I I} k_{\eta \mu \beta \alpha}^{2110}+\frac{1}{2} E_{h i j k}^{I I} k_{\alpha \beta \eta \mu}^{1122}+\frac{1}{2} E_{j k h i}^{I I} k_{\eta \mu \alpha \beta}^{1122}+\frac{1}{2} E_{j k h i}^{I I I} k_{\eta \mu \alpha \beta}^{1111}+ \\
& \left.+\frac{1}{2} E_{h i j k}^{I V} k_{\alpha \beta \eta \mu}^{0022}+\frac{1}{2} E_{j k h i}^{I V} k_{\eta \mu \alpha \beta}^{0022}\right) \bar{d}_{j \eta} \bar{d}_{k \mu}
\end{aligned}
$$

The incremental-iterative procedure was implemented based on (i) Newton-Raphson's method and (ii) a load or arc-length control strategy (both strategies were implemented). The numerical integration is performed using the Legendre-Gauss Quadrature with three-point integration along $x$ (equation (12)) and y (equations (6.1)-(8.13)) the integration along $z$ (though thickness) is done analytically.

\subsection{Cross-section analysis}

The GBT cross-section analysis involves a lengthy set of fairly complex operations which has been reported in the literature, with emphasis on the recent works of Gonçalves et al. (2014) and Bebiano et al. (2015) - the interested reader can find detailed acounts in these references, which provide the fundamentals of the procedure implemented in version 2.0 of code GBTUL (Bebiano et al. 2016). The deformation modes obtained can be divided into three main sets/families, namely the (i) conventional (or Vlasov modes), (ii) shear modes and (iii) transverse extensions modes. These deformation mode sets are also adopted in this work, together with an additional family of quadratic transverse extension modes, which play an essential role in capturing the Poisson effects that develop in the deformed configurations occurring in the advanced post-buckling stages, which involve heavy crosssection in-plane deformation and, therefore, in precluding the determination of overly stiff solutions (Gonçalves \& Camotim 2012) - the aforementioned Poisson effects will be addressed in Section 4.

Figure 2 shows the GBT nodal discretization adopted in this work, which involves 21 nodes (6 natural and 15 intermediate -3 in the top/compressed flange, 1 in the bottom/tensioned flange and 11 in the web ${ }^{5}$ ), and leads

\footnotetext{
5 Note that the post-buckling behavior determined with less web intermediate nodes was found to be excessively stiff (see Section 4.3) - it may be possible to obtain accurate results with less web intermediate nodes if unequal-width wall segments are adopted, making it possible to consider more intermediate nodes in the web compressed region than in tensioned one. However, such a strategy is yet to be attempted.
} 
to (i) 23 conventional modes (4 global, 2 distortional and 17 local) - modes 1-23 (the most relevant ones are shown in Fig. 2), (ii) 20 shear modes (5 global and 15 local) - modes 24-43 (see Fig. 2), (iii) 20 linear transverse extension modes (1 global isotropic, 4 global deviatoric and 15 local) - modes 44-63 (see Fig. 2) and (iv) 20 "quadratic" transverse extension modes (modes 64-83 - see Fig. 2), totaling 83 (sequentially numbered) deformation modes ${ }^{6}$. It should be mentioned that the number of deformation modes considered in this work is almost twice that required to analyze lipped channel columns experiencing L-D interaction ${ }^{7}$ - this is because of the lack of symmetry of the beam cross-section deformation patterns, which did not occur in the columns.

\section{GBT Buckling Behavior - Beam Geometry Selection}

In order to study the mechanics of the geometrically non-linear behavior of lipped channel beams experiencing L-D interaction, it is indispensable to begin by selecting beams geometries (cross-section dimensions and lengths) prone to this coupling phenomenon. Based on previous works, this procedure can be obtained on the sole basis of the closeness between the local $\left(M_{c r L}\right)$ and distortional $\left(M_{c r D}\right)$ critical buckling moments (a necessary condition ${ }^{8}$ ). Therefore, in order to shed new light on this interactive post-buckling behavior, three simply supported beams are identified, namely (i) one experiencing "true L-D interaction", with $R_{D L}=M_{c r D} / M_{c r} \approx 1.00$, and (ii) two affected by "secondary bifurcation L-D interaction", which can be either "local" $\left(R_{D} \approx 0.60\right)$ or "distortional" $\left(R_{D L} \approx 1.60\right)$ - in all of them local buckling is triggered by the compressed (top) flange. Table 1 gives the cross-section dimensions $\left(b_{w}, b_{f}, b_{1}, t\right.$-web-flange-lip widths and wall thickness) of the three steel ( $\left.E=210 \mathrm{GPa}, v=0.3\right)$ beams selected. On the other hand, Figs. 3(a)-(c) show curves providing the variation of $M_{c r}$ (critical buckling moment) with the beam length $L$ (logarithmic scale) for the lipped channel simply supported beams - marked on each of these curves is the selected length adopted to ensure the desired interaction "level". The critical local and distortional buckling moments, as well as the corresponding buckling mode half-wave numbers ( $n_{L}$ and $n_{D}$ ) are also given in Table 1 (between brackets) - moreover, the local and distortional buckling mode shapes are depicted in Figs. 3(a)-(c)). In order to preclude the interaction with global (flexural-torsional) buckling, it was ensured that the critical global buckling moment is much higher than its local and distortional counterparts (see Table 1).

Table 1: Simply supported lipped channel beam geometries selected, corresponding local and distortional buckling moments, and buckling mode critical half-wave numbers (dimensions in $\mathrm{mm}$ and moments in $\mathrm{kNcm}$ )

\begin{tabular}{cccccccccc}
\hline Beam & $b_{w}$ & $b_{f}$ & $b_{l}$ & $t$ & $L$ & $M_{c r D}$ & $M_{c r L}$ & $M_{c r G}$ & $R_{D L}$ \\
\hline B1 & 110 & 75 & 12.5 & 1.050 & 500 & $220.9(1)$ & $217.0(7)$ & $9796(1)$ & 1.02 \\
B2 & 130 & 70 & 16.0 & 0.935 & 500 & $331.2(1)$ & $207.4(7)$ & $10103(1)$ & 1.60 \\
B3 & 150 & 95 & 10.0 & 1.350 & 600 & $285.6(1)$ & $473.6(6)$ & $21223(1)$ & 0.60 \\
\hline
\end{tabular}

\section{GBT Post-Buckling Behavior of Beams Experiencing L-D Interaction}

The selected simply supported beams are now used to investigate the influence of L-D interaction on their postbuckling behavior, by means of GBT GNIA analyses, with the objective of shedding fresh light on the mechanics of the three aforementioned L-D interaction types. The results of beam B1, experiencing "true L-D interaction" (TI) are addressed first, in Section 4.1, followed by those concerning beams B2 and B3, undergoing "secondary distortional-bifurcation L-D interaction" (SDI - Section 4.2) and "secondary local-bifurcation L-D interaction" (SLI Section 4.3) - this sequence corresponds to a decreasing relevance of the L-D interaction effects (Martins et al. 2016c). All the beams analyzed contain critical-mode initial geometrical imperfections with small amplitudes

\footnotetext{
6 It should be noted that none of the previously developed GBT geometrically non-linear formulations employed the set of deformation modes considered in this work. This is because the cross-section analysis procedure employed here was not yet available - alternative (less sophisticated) cross-section analysis procedures were adopted.

7 Although these results are not yet published, they were very recently presented at the "European Congress on Computational Methods in Applied Sciences and Engineering" (ECCOMAS'2016), which took place in Crete on June 5-10, 2016.

8 However, as will be shown later, this condition is not sufficient in the columns affected by "secondary bifurcation L-D interaction".
} 

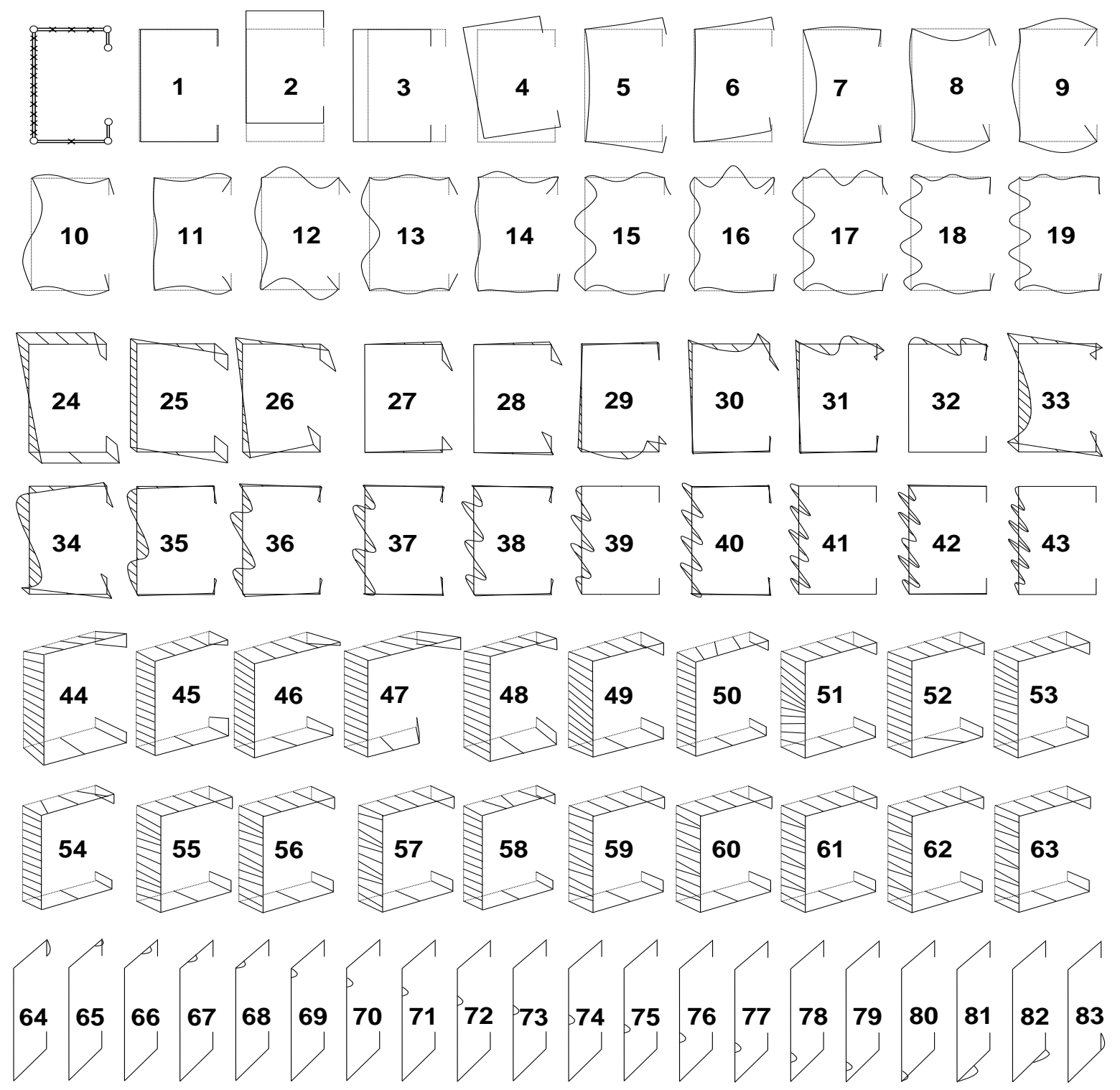

Figure 2: GBT lipped channel (i) nodal discretization, (ii) in-plane deformed configurations of the most relevant conventional and all transverse extension deformation modes, and (iii) warping displacement profiles of all shear deformation modes

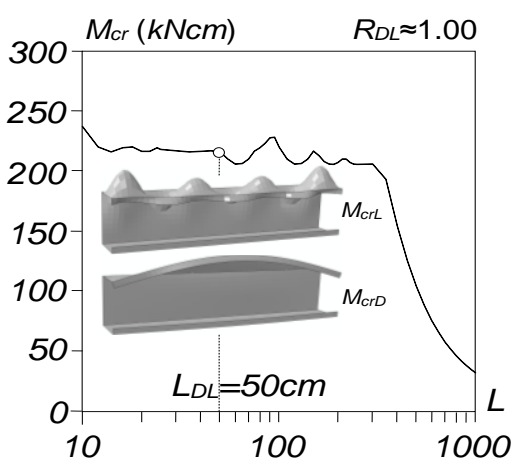

(a)

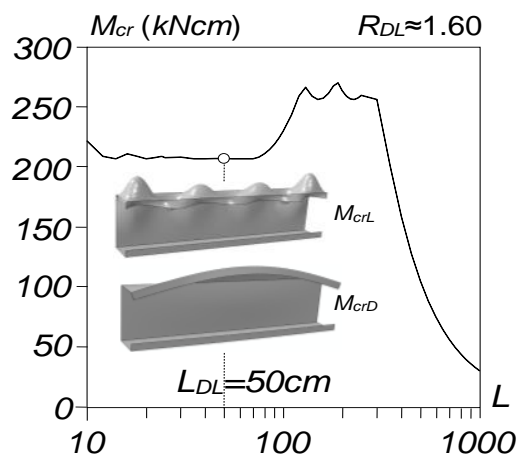

(b)

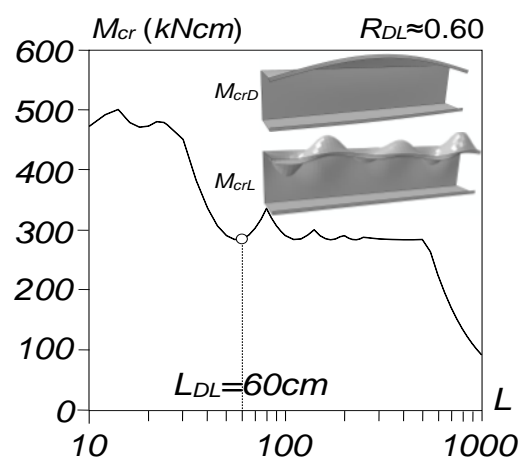

(c)

Figure 3: (a) $R_{D L}=1.00$, (b) $R_{D L}=1.60$ and (c) $R_{D L}=0.60$ beam $M_{c r}$ vs. $L$ curves, selected lengths and critical mode shapes 
( $10 \%$ of the wall thickness $t$ ), obtained from preliminary buckling analyses. All the GBT results are compared with values provided by shell finite element analyses (SFEA) carried out in ABAQUS (Simulia 2008), employing models previously adopted in a study on beam L-D interaction (Martins et al. 2016c). It is worth noting that, in order to achieve accurate post-buckling results, (i) fairly fine cross-section and longitudinal discretizations must be adopted, and (ii) a quite high number of deformation modes must be included in the GNIA - these three inputs are much more demanding than those required to perform accurate buckling analyses.

\subsection{True L-D Interaction}

Fig. 4 shows the equilibrium path $M / M_{c r} v s$. $\left(v+v_{0}\right) / t$ ( $v$ is the mid-span top flange-lip corner vertical displacement caused by the applied moment $M$, i.e., excluding the initial imperfection value $v_{0}=0.1 t$ ), obtained with an arclenght crontrol strategy for the simply supported lipped channel beam B1 under uniform major-axis bending the initial geometrical imperfection shape adoped is also shown and consists of 7 local half-waves (akin to the critical local buckling mode). Naturally, the modal participations factors provided by the GBT buckling analysis indicate major contributions from the local modes: 7-26.5\%, 8-31.3\%, 9-23.9\%, 10-3.3\%, 11-3.1\%, 12-4.8\%, 13-2.0\% - the remaining (minute) contributions concern other local modes and the two distortional modes ${ }^{9}$. In order to assess the relevance of the different deformation modes families to the beam post-buckling behavior, several "approximate analyses" were performed, namely those including the most relevant (i) conventional and linear transverse extension modes (1-21+40-57), (ii) conventional, shear and linear transverse extension modes (1-57) - both considering 8 finite elements - and (iii) conventional, shear and transverse extension (linear and quadratic) modes, considering 8 and 24 finite elements. On the other hand, Figs. 5(a)-(c) and 6(a)-(f) display the evolution, along the most accurate equilibrium path in Fig. 4 , of the mid-top flange transverse displacement $(w(x))$ profiles due to various mode sets. While Figs. 5(a)-(c) concern the initial post-buckling stages and show the contributions from modes 5+6, 7-21 and 1-57 $\left(w_{5+6}(x), w_{7-21}(x)\right.$ and $w_{1-57}(x) \equiv w(x)$, respectively), Figs. 6(a)-(f) concern mainly the most advanced post-buckling stages and show contributions from modes 2, 4, 5+6, 7-21, 40-57 and 1-57 ( $w_{2}(x)$, $w_{4}(x), w_{5+6}(x), w_{7-21}(x), w_{40-57}(x)$ and $w_{1-57}(x) \equiv w(x)$, respectively), i.e., six combinations of all the modes contributing to the mid-top flange transverse displacement - note that the contributions of modes $\mathbf{3}$ and 58-75 are null, since the former is an horizontal (normal to the web) translation and the latter have null values at each node. Finally, Fig. 7 shows beam deformed configurations at several post-buckling stages, namely $M / M_{c}=0.50,0.75,1.00$ and 1.18 (peak moment) - the amplifications adopted for these configurations are indicated between brackets. The observation of these results prompts the following remarks:

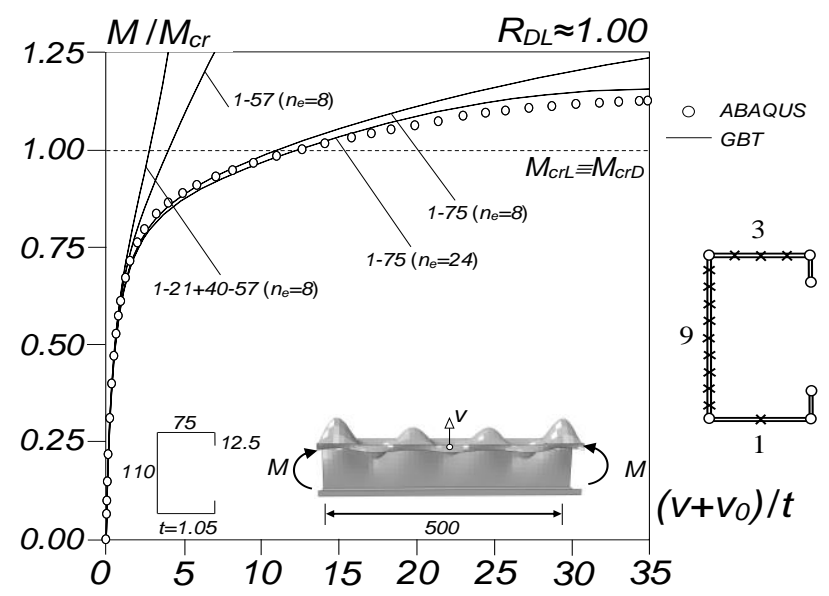

Figure 4: GBT post-buckling equilibrium paths of the $R_{D L}=1.02$ lipped channel beam undergoing "true L-D interaction" obtained with local initial geometrical imperfections and different deformation mode sets and/or finite element numbers

\footnotetext{
9 Note that, for the simply supported beams analyzed in this work, all modal amplitude functions are sinusoidal (with 7 half-waves in this case).
} 


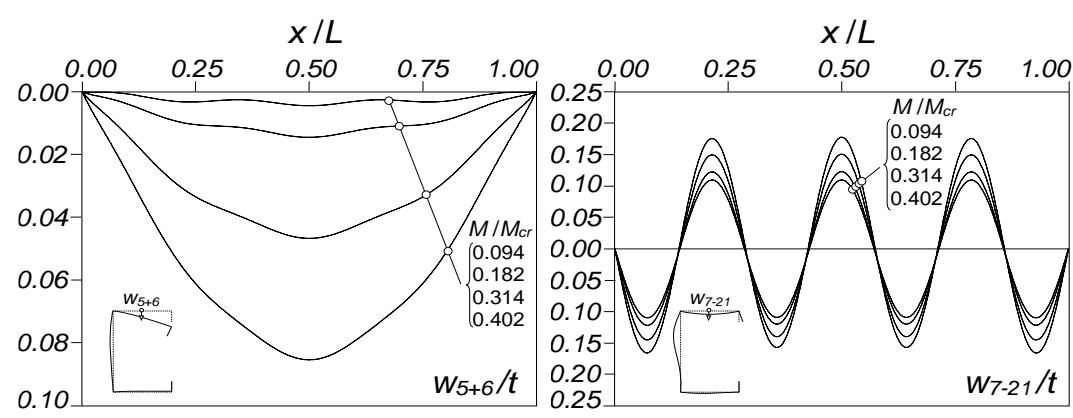

(a) (b)

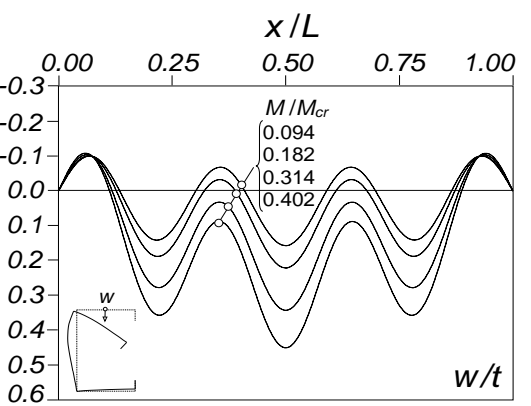

(c)

Figure 5: Evolution of the $R_{D L}=1.02$ beam top flange transverse bending displacement profiles due to deformation modes (a) 5+6 $\left(w_{5+6}(x)\right),\left(\right.$ b) 7-21 $\left(w_{7-21}(x)\right)$ and (c) 1-75 $\left(w_{1-75}(x) \equiv w(x)\right)$ - early loading stages

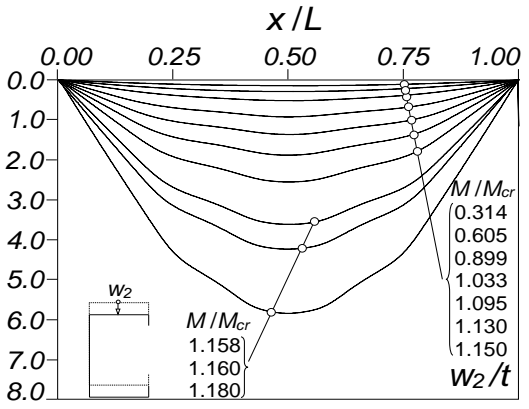

(a)

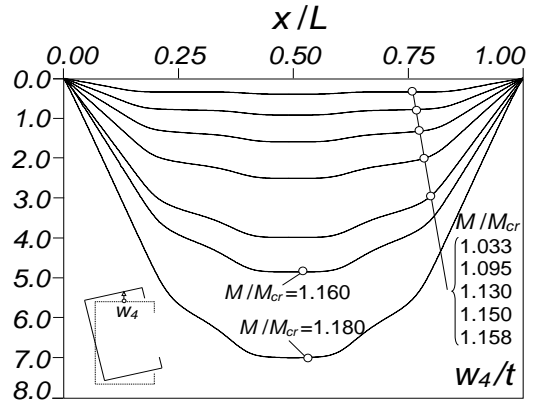

(b)

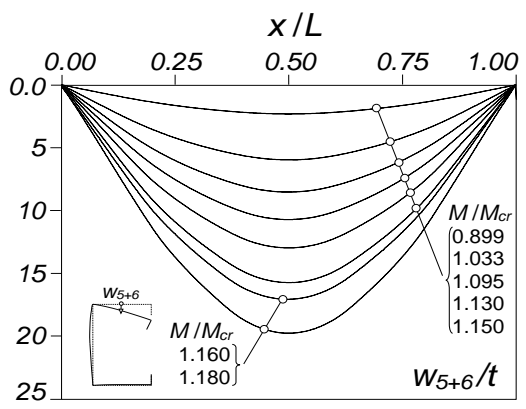

(c)

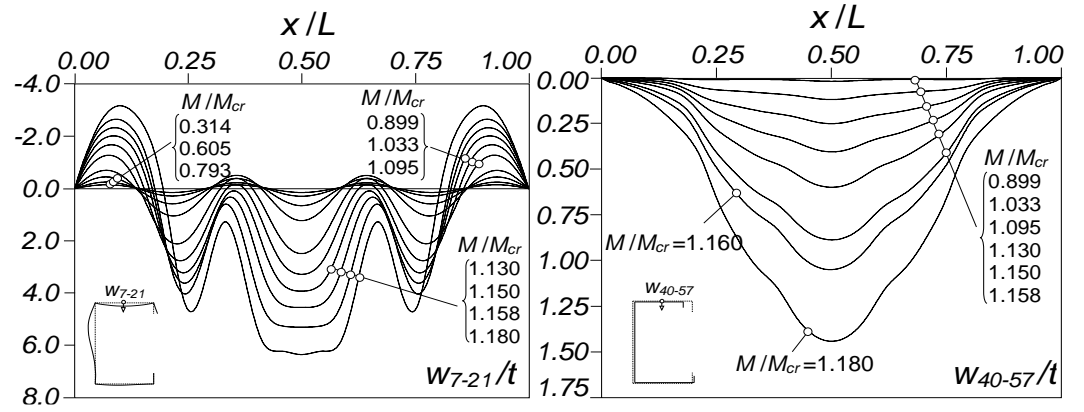

(d)

(e)

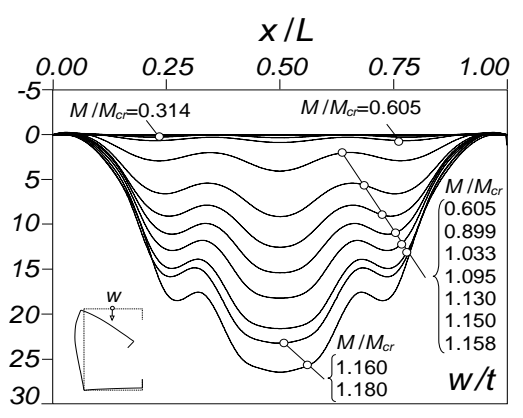

(f)

Figure 6: Evolution of the $R_{D L}=1.02$ beam mid-top flange transverse bending displacement profiles due to deformation modes (a) $2\left(w_{2}(x)\right)$, (b) $4\left(w_{4}(x)\right)$, (c) 5+6 ( $\left.w_{5+6}(x)\right)$, (d) 7-21 (w7-21 $\left.(x)\right),(\mathrm{e})$ 40-57 ( $\left.w_{40-57}(x)\right)$ and (f) 1-75 $\left(w_{1-75}(x) \equiv w(x)\right)$

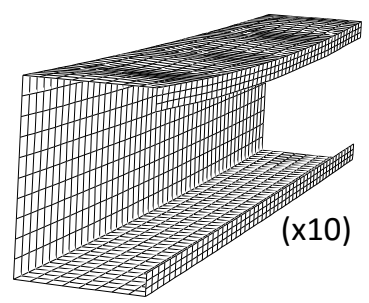

$M / M_{c r}=0.50$

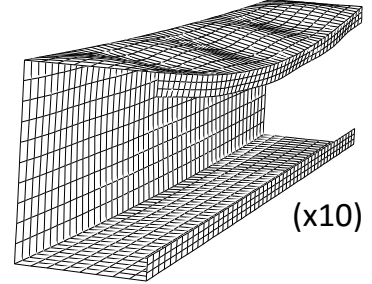

$M / M_{c r}=0.75$

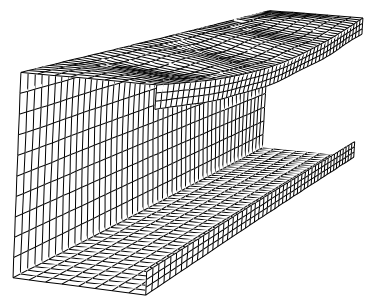

$M / M_{c r}=1.00$

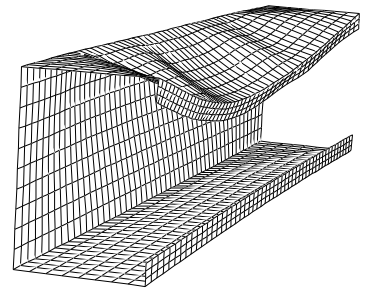

$M / M_{c r}=1.18$

Figure 7: ABAQUS $R_{D L}=1.02$ beam deformed configurations at $M / M_{c r}=0.50 ; 0.75 ; 1.00 ; 1.18$ (peak moment)

(i) The GBT equilibrium path obtained with all the conventional, shear and transverse extension modes, and 24 finite elements, is very close to the path provided by the ABAQUS SFEA up to $\left(v+v_{0}\right) / t=35$, despite the disparity of the d.o.f. numbers employed in the two post-buckling analyses - the small difference between the two curves could be reduced/eliminated if 11 intermediate nodes were considere in the web (instead of 9). 
(ii) Fig. 4 shows the relevance of the quadratic transverse extension modes in capturing accurately the beam post-buckling behavior, thus confirming the findings of Gonçalves \& Camotim (2012). In fact, when these modes are excluded from the analysis (solution 1-57 in Fig. 4), accurate results are obtained only up to fairly early post-buckling stages $\left(M / M_{c r}=0.75\right)$ - after that, membrane locking effects lead to an overly stiff solution.

(iii) The (most accurate) equilibrium path shown in Fig. 4 exhibits a fairly rapid stiffness erosion/degradation until an elastic limit point is reached for $M / M_{c r}=1.18$ - in fact, the detrimental effect of true L-D interaction precludes reaching higher strengths (the peak moment is close to the $M_{c r}-$ about $1.18 M_{c r}$ in this particular case). Higher post-critical strengths are reached in the beams affected by the other two L-D interaction types - see Figs. 8 and 12, concerning beams B2 (SDI) and B3 (SLI).

(iv) Since the local initial geometrical imperfection exhibits 7 half-waves, it is logical to expect the mid-top flange transverse displacement profile $w_{7-21}(x)\left(\mathrm{iv}_{1}\right)$ to retain this shape as loading progresses (see Fig. $\left.5(\mathrm{~b})\right)$ and $\left(\mathrm{iv}_{2}\right)$ to coincide with $w(x)$ at the early loading stages (Fig. 5(c)) - indeed, $w_{7-21}(x)$ is a mere amplification of the initial imperfection. Conversely, $w_{5-6}(x)$ (Fig. 5(a)) shows very clearly the gradual deformed configuration change from 7 half-waves (akin to the initial imperfection - e.g., for $M / M_{c r}=0.094$ ) to a single distortional halfwave (akin to the distortional critical buckling mode) - e.g., at $M / M_{c r}=0.402$ this displacement profile is already fairly close to a sinusoid. Moreover, at this stage $w(x)$ reflects the combination of these two deformation sources, providing clear evidence of L-D interaction - the gradual emergence, at early loading stages, of deformation patterns akin to the critical local and distortional buckling modes (see all the deformed configurations displayed in Fig. 7) characterizes the so-called "true L-D interaction" (TI).

(v) As shown in Fig. 6, for higher loading levels the dominant contribution to $w(x)$ comes from the distortional modes $\mathbf{5 + 6}$ (see Figs. 6(c) and (f)), a feature typical of L-D interaction (regardless of the type). Concerning the contribution of the local modes (see Fig. $6(d)$ ), it is interesting to notice that, for $M / M_{c r}>0.697, w_{7-21}(x)$ progressively moves away from the initial 7 equal-shaped half-waves - these half-waves become unequal and more pronounced (downwards) in the beam central region. On the other hand, it is also worth noting the relevance of the contributions from modes 2 and $4^{10}$ - such contributions are of the same order of magnitude as those exhibited by modes 7-21 (see Figs. 6(a)-(b) and (d), for $\left.M / M_{c r}=1.180\right)$. It is still worth mentioning that the emergence of mode 3 (linked to mode $\mathbf{4}$ ), which is neither directly caused by the loading (major-axis bending) nor akin to the competing (local and distortional) buckling modes, stems from the considerable stress redistribution occurring in the cross-section compressed/top half at the advanced post-buckling stages and is, most likely, responsible for the beam rapid stiffness erosion/degradation ${ }^{11}$. As far as the contribution of the (linear) transverse extensions modes to $w(x)$ (see Fig. 6(e)), the main sources are the global isotropic mode $\mathbf{4 0}$ and global deviatoric modes $\mathbf{4 3}$ and $\mathbf{4 4}$ (mostly the latter). Although this contribution is quantitatively very small, with respect to the remaining ones shown in Fig. 6, recall that the inclusion of the transverse extension modes in the beam GNIA is absolutely essential to obtain accurate results (see Fig. 4) ${ }^{12}$.

\subsection{Secondary Distortional-Bifurcation L-D Interaction}

Since the results addressed in this section, which concern beam B2, are similar to those reported in Section 4.1 (for beam B1), their presentation and discussion are abbreviated as much as possible. Fig. 8 shows $M / M_{c r L} v s .\left(v+v_{0}\right) / t$ equilibrium paths, obtained again with an arc-length control strategy and concerning a beam with a 7 half-wave local initial geometrical imperfection, akin to the critical buckling mode - a GBT buckling analysis showed that the most relevant contributions are from local deformation modes: 7-27.1\%, 8-26.5\%, 9-24.0\%, 10-5.6\%, 11-1.9\%,

\footnotetext{
${ }^{10}$ It is worth noting that modes $\mathbf{3}$ and $\mathbf{4}$ are linked and, therefore, their emergence is simultaneous and their longitudinal amplitude functions share the same shape. In this case, the contribution of modes $\mathbf{3}$ points towards the web and that of mode $\mathbf{4}$ is counterclockwise. Since mode 3 does not contribute to $w(x)$, it is not shown in Fig. 6 .

${ }^{11}$ This same behavioral feature occurs also in beams B2 and B3, addressed in Sections 4.2 and 4.3, although to a smaller extent (less rigid-body motion) - probably, this fact explains the slower stiffness erosion/degradation, making it possible to achieve higher post-critical strengths.

12 This assertion concerns both the linear and quadratic transverse extension modes, even if the latter do not contribute to $w(x)$.
} 
12-6.4\%, 13-4.9\% (participations similar to those of beam B1). Once more, the various equilbrium paths are determined with various deformation mode sets: (i) conventional+linear transverse extension modes (1-23+44-63) and (ii) conventional+shear+linear transverse extension modes (1-63), considering 8 finite elements in both cases, and (iii) conventional+shear+transverse extension (linear and quadratic) modes, considering 8 and 30 finite elements. As before, Figs. 10(a)-(f) and 11 show, respectively, (i) the evolution, along the most accurate equilibrium path, of the mid-top flange transverse displacement profiles associated with modes 2, 4, 5+6, 7-23, 44-63 and 1-83 $\left(w_{2}(x), w_{4}(x), w_{5+6}(x), w_{7-23}(x), w_{44-63}(x)\right.$ and $\left.w_{1-83}(x) \equiv w(x)\right)$, and (ii) the beam deformed configurations at $M / M_{c r}=0.75,1.00,1.25$ and 1.38 (peak moment)) - Figs. 9(a)-(c) plot the evolution $w_{2}(x), w_{4}(x)$ and $w_{5+6}(x)$, in the early loading stages. The observation of the results presented in these figures leads to the following conclusions:

(i) The general comments made in items (i) and (ii) of Section 4.1 remain valid. However, it should be noted that, due to a more refined cross-section (nodal) and longitudinal discretization ${ }^{13}$, the GBT results now coincide with the SFEA ones. Moreover, the gradual stiffness erosion is slightly less pronounced in beam B2, thus leading to the occurrence of the elastic limit point at a higher applied loading level $\left(M / M_{c L}=1.38\right)$.

(ii) As expected, in the early loading stages $w(x)$ (Fig. 9(c)) has dominant contributions from (ii 1 ) the local modes 7-23 (Fig. 9(b)) and (ii 2 ) major-axis flexure (mode 2 - Fig. 10(a)). Like in beam B1, Fig. 9(a) shows clearly the $W_{5-6}(x)$ shape switch from 7 half-waves (akin to the initial imperfection - e.g., for $M / M_{c r l}=0.094$ ) to a single half-wave (akin to the distortional critical buckling mode - e.g., at $M / M_{c r}=0.622 w_{5-6}(x)$ is already quite close to a perfect sinusoid). This early switch is rather surprising, in view of how far apart the local and distortional critical buckling moments are. Indeed, the $w_{5-6}(x)$ profiles of beams B2 $\left(R_{D L}=1.60\right)$ and B1 $\left(R_{D L}=1.02\right)$ are fairly similar - the half-wave number switch takes place only a bit sooner in the latter ${ }^{14}$. Thus, it is concluded that the beam SDI effects are quite close to the TI ones (i.e., more severe than anticipated).

(iii) As pointed out earlier, once deformations akin to the distortional critical buckling mode emerge, they rapidly become the major source of cross-section in plane deformation (see Fig. 10(c)-(f)) - indeed, for $M / M_{c r}>1.263$ $w_{5-6}(x)$ provides the highest contribution to $w(x)$. Concerning the contributions of the remaining modes,

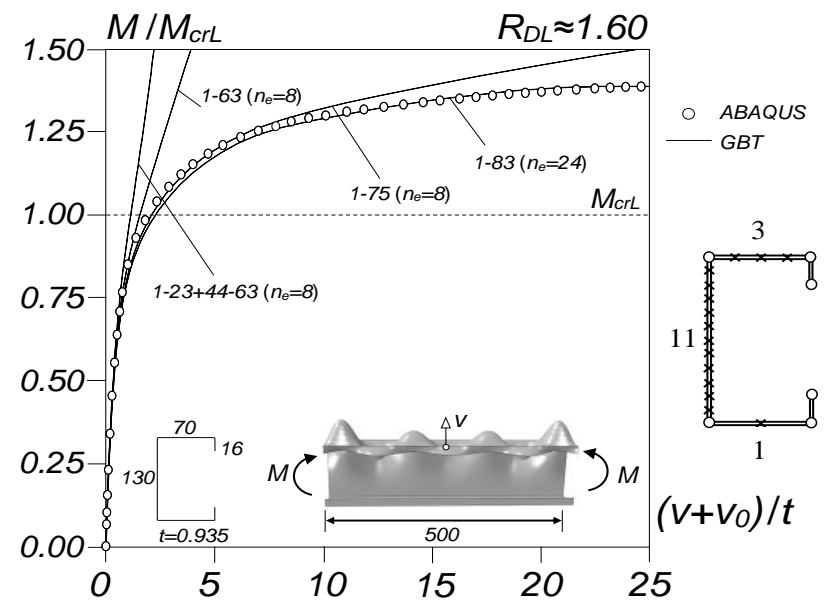

Figure 8: GBT post-buckling equilibrium paths of the $R_{D L}=1.60$ lipped channel beam under "secondary distortional-bifurcation L-D interaction" obtained with local initial imperfections and various deformation mode sets and/or finite element numbers

\footnotetext{
${ }^{13}$ It involves (i) 21 nodes ( 6 natural and 15 intermediate -3 in the top/compressed flange, 1 in the bottom/tensioned flange and 11 in the web) and (ii) 30 finite elements.

${ }^{14}$ This similarity, which was not observed in columns experiencing TI and SDI, is most likely due the amount of local deformation developing in the compressed flange (the wall triggering local buckling), which causes a drop in "distortional stiffness" that leads to a lower distortional critical buckling moment and, therefore, justifies the early emergence of deformations akin to the corresponding buckling mode. In the columns analyzed, local buckling is triggered by the web, therefore, the development of local deformations has little impact on the flange "distortional stiffness" - this picture would probably change if the column local buckling was triggered by the flanges (such a situation was never studied by the authors).
} 


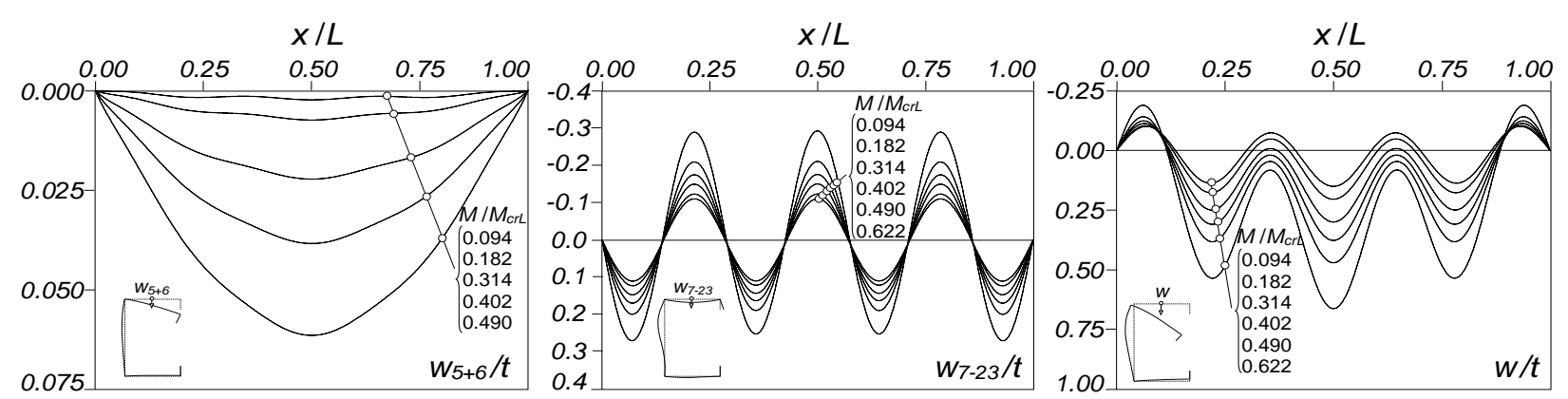

Figure 9: Evolution of the $R_{D L}=1.60$ beam top flange transverse bending displacement profiles due to deformation modes (a) 5+6 $\left(w_{5+6}(x)\right)$, (b) 7-21 $\left(w_{7-21}(x)\right)$ and (c) 1-83 $\left(w_{1-83}(x) \equiv w(x)\right)$ - early loading stages

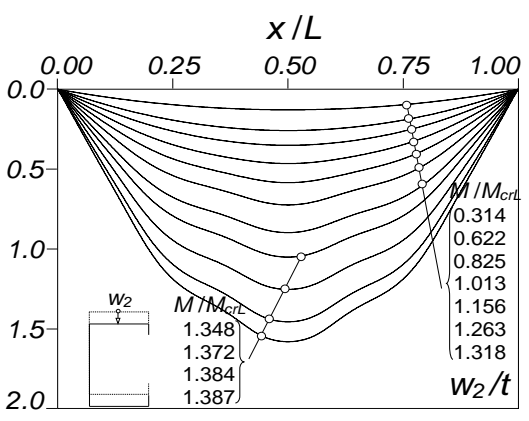

(a)

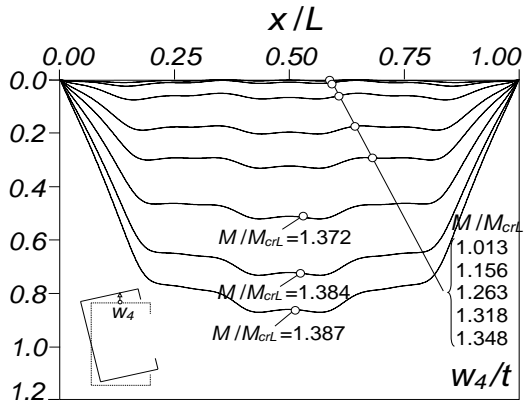

(b)

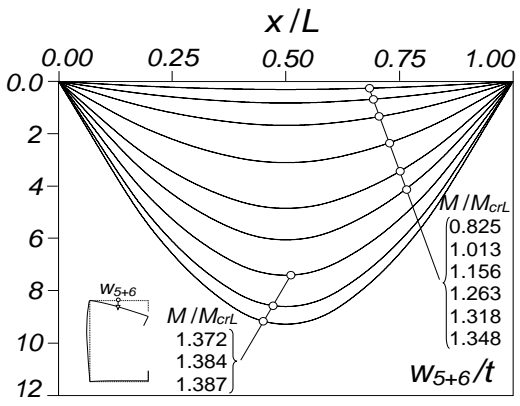

(c)

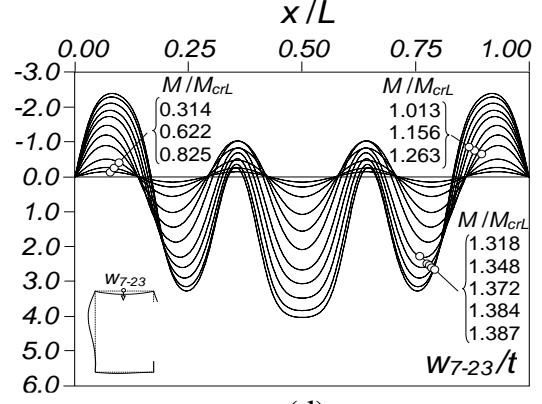

(d)

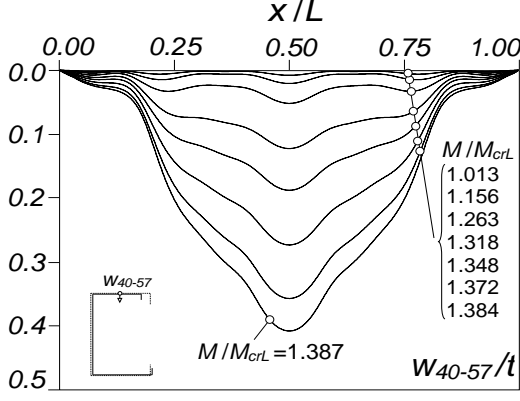

(e)

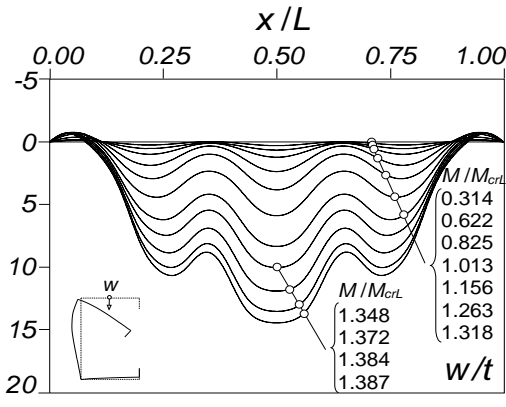

(f)

Figure 10: Evolution of the $R_{D L}=1.60$ beam top flange transverse bending displacement profiles due to deformation modes (a) $2\left(w_{2}(x)\right),(\mathrm{b}) 4\left(w_{4}(x)\right),(\mathrm{c})$ 5+6 $\left(w_{5+6}(x)\right),(\mathrm{d})$ 7-23 $\left(w_{7-23}(x)\right),(\mathrm{e})$ 40-57 $\left(w_{40-57}(x)\right)$ and (f) 1-83 $\left(w_{1-83}(x) \equiv w(x)\right)$

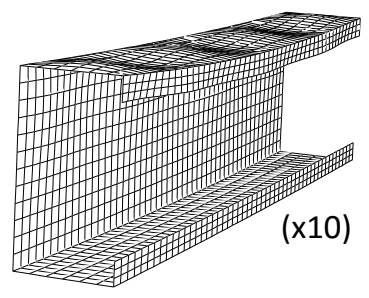

$M / M_{c r L}=0.75$

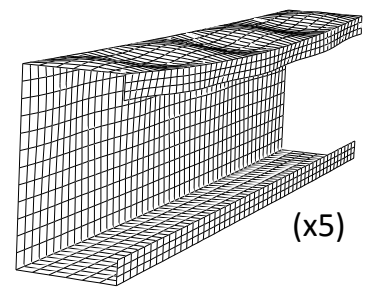

$M / M_{c r L}=1.00$

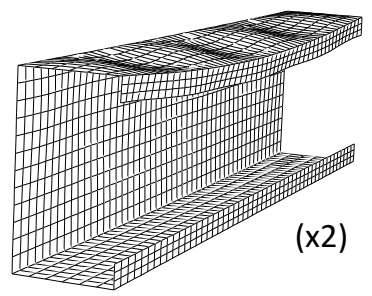

$M / M_{c r L}=1.25$

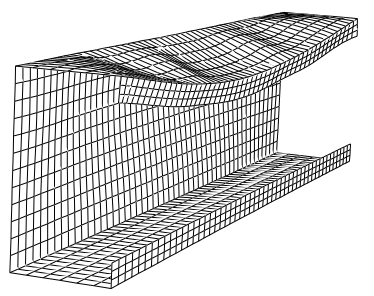

$M / M_{c r L}=1.38$

Figure 11: ABAQUS $R_{D L}=1.60$ beam deformed configurations at $M / M_{c r L}=0.75 ; 1.00 ; 1.25 ; 1.38$ (peak moment)

they follow the same general trends of those obtained for beam B1 - however, there are a few differences. Indeed, $w_{7-23}(x)$ (Fig. 10 (d)) exhibits "more perfect" half-waves that start becoming unequal at $M / M_{c r} \approx 0.622$. On the other hand, the contributions of the rigid-body modes $\mathbf{2}$ and $\mathbf{4}$ (linked to $\mathbf{3}$ ) are smaller than the beam B1 ones - moreover, $w_{4}(x)$ exhibits now slightly more irregular/wavy patterns. 


\subsection{Secondary Local-Bifurcation L-D Interaction}

Fig. 12 shows the beam B3 equilibrium paths $M / M_{c r D}$ vs. $\left(v+v_{0}\right) / t$, now obtained with load/moment control (increments equal to 5 or $7.5 \%$ of $M_{c r D}$, depending on the deformation mode set included in the analysis, up to $1.50 M_{c r D}$ ) and a critical-mode initial geometrical imperfection (single distortional half-wave with inward top flangelip motions). The GBT buckling analysis showed that the critical buckling mode combines major contributions from modes 5-52.3\% and 6-43.0\% - the remaining (minute) contributions are from local modes $(\mathbf{7}-1.4 \%, 8-1.7 \%$ and 9-1.0\%) and also exhibit a single half-wave. Unlike for beams B1 and B2, all the equilibrium paths depicted in Fig. 12 were determined by considering different cross-section and/or longitudinal discretizations and including in the GNIA all the deformation modes available. The discretizations adopted differ only in the intermediate node and finite element (FE) number: (i) 3 flange +3 web nodes and 8 FEs (" $A$ "), (ii) 3 flange +5 web nodes and 8 FEs ("B"), (iii) 3 flange +7 web nodes and 8 FEs ("C $C$ "), (iv) 3 top flange +1 bottom flange +9 web nodes and 8 FEs ("D") and (v) 3 top flange +1 bottom flange +9 web nodes and 18 FEs ("E"). Moreover, Figs. 13(a)-(f) depict again the mid-top flange transverse displacement profiles due to the contributions of modes 2, 4, 5+6, 7-21, 40-75 and 1-75 $\left(w_{2}(x)\right.$, $\left.w_{4}(x), w_{5+6}(x), w_{7-21}(x), w_{40-57}(x), w_{1-75}(x) \equiv w(x)\right)$. Finally, Fig. 14 shows the beam deformed configurations at four postbuckling stages $\left(M / M_{c r D}=0.75,1.00,1.25,1.45\right)$. These results make it possible to draw the following conclusions:

(i) Fig. 12 provides clear confirmation of the pivotal role played by the number of web intermediate nodes in the accuracy of the results obtained, particularly after the emergence and development of L-D interaction. Indeed, the accuracy of the GBT equilibrium path continuously improves as the number of web intermediate grows (between solutions " $A$ " and " $D$ ") ${ }^{15}$. For the most refined web discretization, the inclusion all the deformation modes available (conventional, shear and transverse extension - linear and quadratic) and the consideration of 18 finite elements provides a rather accurate solution up to $M / M_{c r D}=1.50$ - solution " $E$ ".

(ii) Fig. 12 clearly shows that the stiffness of the beam B3 equilibrium path drops significantly at $M / M_{c r D} \approx 1.20$, which is the applied loading level corresponding to the emergence of local deformations (see Fig. 13(d)).

(iii) For $M / M_{c r D}<1.20$, the beam post-buckling behavior is typically distortional, i.e., $w(x)$ exhibits a single halfwave (see Fig. 13(f)) combining (iii $1_{1}$ ) a dominant contribution from the distortional modes $\mathbf{5 + 6}$ (Fig. 13(c)) with (iiii) contributions from mode $\mathbf{2}$ (major-axis flexure - Fig. 13(a)) and, to a smaller extent, from the local (7-21 - Fig. 13(d)) and transverse extension (40-57 - Fig. 13(e)) modes - there are also minute contributions from the linked modes $\mathbf{3}$ (minor-axis flexure pointing towards de web) and $\mathbf{4}$ (torsion - Fig. 13(b)), which are

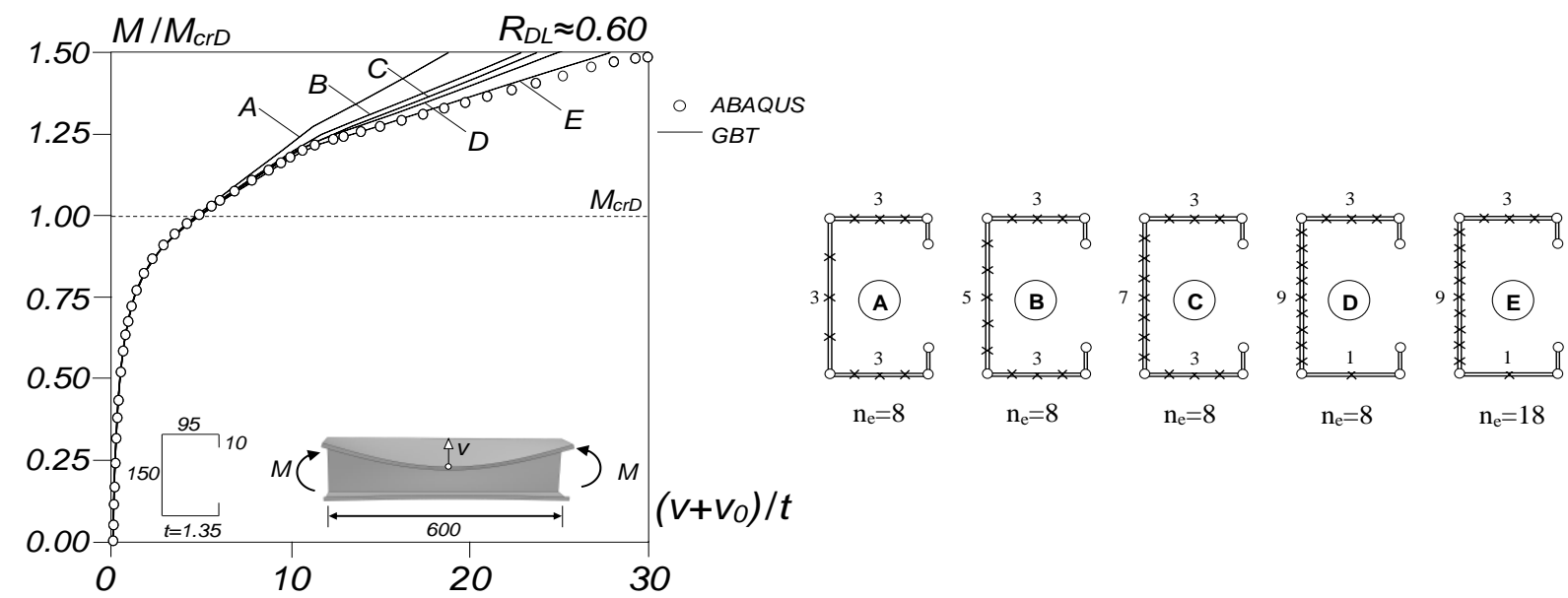

Figure 12: GBT post-buckling equilibrium paths of the $R_{D L}=0.60$ lipped channel beam under "secondary local-bifurcation L-D interaction" obtained with distortional initial imperfections and different discretizations and/or finite element numbers

\footnotetext{
${ }^{15}$ In columns undergoing L-D interaction, it was found that accurate equilibrium paths can be obtained with only 3 web intermediate nodes - the fact that web deformation is now much more complex (double curvature and lack of symmetry) explains the need for a (much) more refined web discretization.
} 


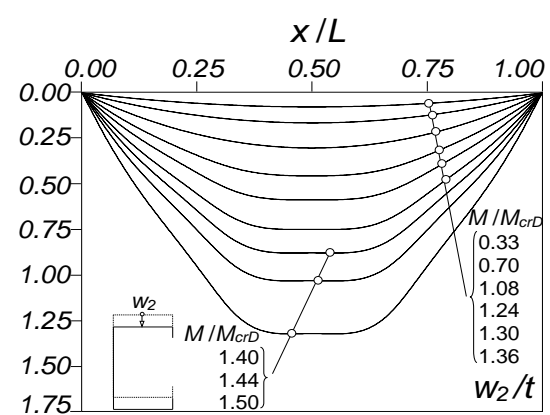

(a)

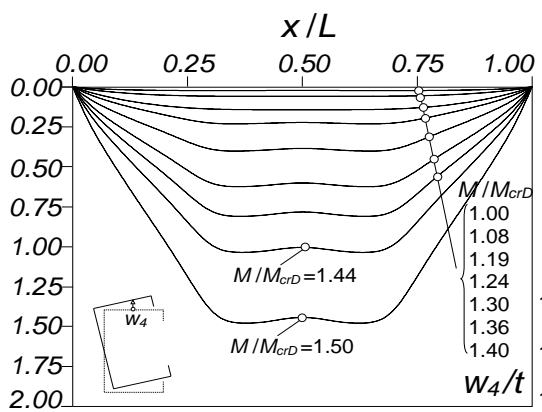

(b)

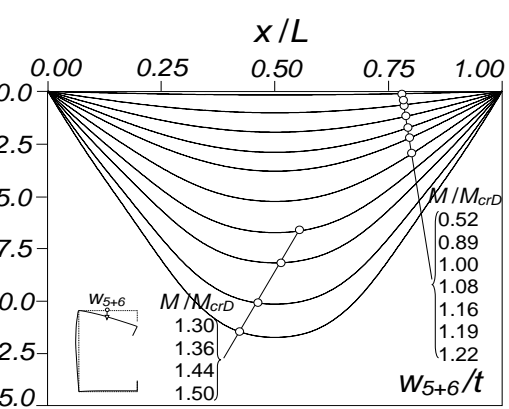

(c)

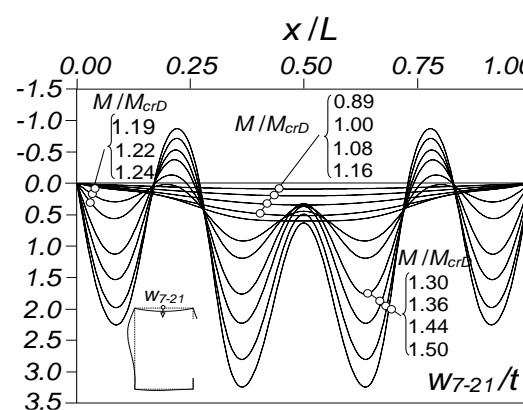

(d)

$x / L$ $x / L$

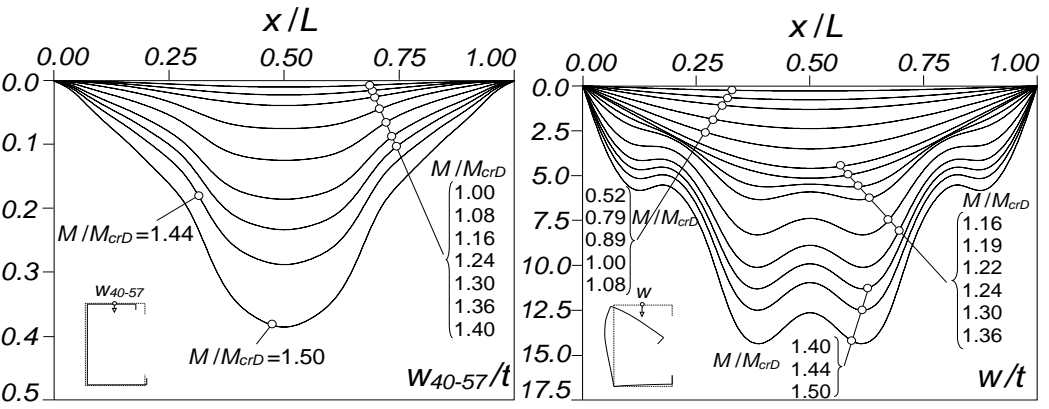

(e)

(f)

Figure 13: Evolution of the $R_{D L}=0.60$ beam top flange transverse bending displacement profiles due to deformation modes (a) $2\left(w_{2}(x)\right)$, (b) $4\left(w_{4}(x)\right)$, (c) 5+6 $\left(w_{5+6}(x)\right)$, (d) 7-21 (w7-21 $\left.(x)\right)$, (e) 40-57 (w40-57 $\left.(x)\right)$ and (f) 1-75 (w1-75 $\left.(x) \equiv w(x)\right)$

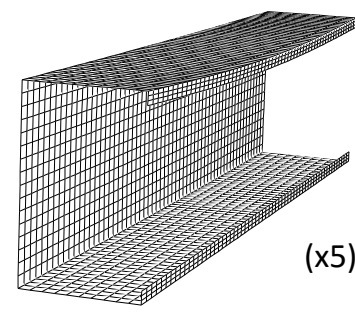

$M / M_{c r D}=0.75$

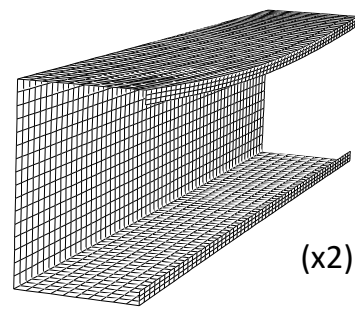

$M / M_{c r D}=1.00$

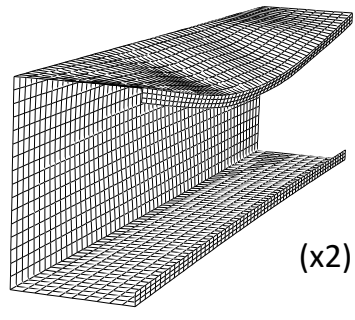

$M / M_{c r D}=1.25$

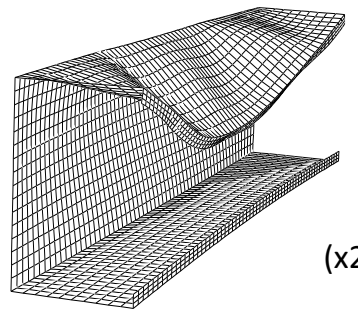

$M / M_{c r D}=1.45$

Figure 14: ABAQUS $R_{D L}=0.60$ beam deformed configurations at $M / M_{C r D}=0.75 ; 1.00 ; 1.25 ; 1.45$

"flattened" in the beam central region. The above post-buckling behavior is clearly illustrated by the first two deformed configurations shown in Fig. 14, concerning the loading levels $M / M_{c r D}=0.75$ and $M / M_{c r D}=1.00$.

(iv) However, when the applied loading level increases beyond $M / M_{c r D}>1.20$ the $w(x)$ shape gradually switches from a single half-wave to seven half-waves, due to the emergence and development of local deformations with seven half-waves (i.e., akin to the second local buckling mode - see the evolution of $w_{7-21}(x)$ in Fig. 13(d)). The (apparently) surprising emergence of local deformations not akin to the critical local buckling mode, which contains six half-waves (see Table 1 ), is due to the fact that (iv $\left.v_{1}\right)$ the first two local buckling modes are associated with virtually coincident buckling moments $(473.58 \mathrm{vs} .473 .89 \mathrm{kNcm})^{16}$ and $\left(\mathrm{iv}_{2}\right)$ the latter is symmetric, like the (single half-wave) critical distortional buckling mode - this shared symmetry favors the interaction between the two buckling modes. This is confirmed by observing the growing visibility of a seven half-wave local contribution to $w(x)$ shape, as the applied loading increases beyond $M / M_{c r D}=1.20$ - see also the deformed configurations corresponding to $M / M_{c r D}=1.25$ and $M / M_{c r D}=1.45$ depicted in Fig. 14 .

\footnotetext{
${ }^{16}$ Most likely due to this extreme buckling moment closeness, numerical difficulties were encountered to determine correct equilibrium states (in the sense of being similar to those provided by the SFEA) in the vicinity of $M / M_{c r D}=1.20$. It was necessary to adopt very small load increments to obtain the correct displacement longitudinal profiles.
} 
The emergence of a deformation pattern akin to the critical local buckling mode, at fairly advanced loading stages, characterizes the so-called "secondary local-bifurcation L-D interaction" (SLI). Like in columns, the beam SLI effects are less pronounced than the TI and SDI ones.

(v) Concerning the other contributions to the evolution of $w(x)$, namely those from modes 2 and 4 (linked to 3 ), their configurations remain practically unchanged - only the amplitudes increase ${ }^{17}$.

\section{Conclusion}

A GBT-based numerical investigation concerning the geometrically non-linear (elastic) post-buckling behavior of simply supported lipped channel beams under uniform bending and affected by three types of L-D interaction, namely (i) "true L-D interaction", (ii) "secondary distortional-bifurcation L-D interaction" and (iii) "secondary localbifurcation L-D interaction", was reported. Particular attention was paid to the structural interpretation of the behavioral features stemming from the L-D interaction effects, aimed at shedding fresh light on the mechanics of this coupling phenomenon. The results presented and discussed consisted of equilibrium paths and plots providing the evolution, along those equilibrium paths, of the beam (i) relevant displacements profiles and (ii) deformed configurations. They were validated through the comparison with values yielded by ABAQUS SFEA.

After presenting, in detail, the main steps involved in the development of a novel GBT formulation to analyze the elastic non-linear behavior of thin-walled members, GBT buckling analyses were employed to obtain the geometry of three simply supported lipped channel beams experiencing the three types of L-D interaction under consideration in this work - local buckling was triggered by the compressed flange in all of them, which corresponds to case most encountered in practice. Then, the results obtained by means of the GBT geometrically non-linear imperfect analysis (GNIA) of the three beams were presented and discussed, taking advantage of the GBT modal features - the compressed flange transverse bending behavior was the main vehicle used to acquire in-depth knowledge on the mechanics of L-D interaction.

Among the various conclusions drawn from this investigation, the following deserve to be specially mentioned:

(i) In spite of the different mechanics exhibited by the three beams analyzed in this work, experiencing different types of L-D interaction (TI, SDI and SLI), all of them share a common trait: occurrence of deformation patterns akin to both the local and distortional critical buckling modes, whose coupling leads to a post-buckling strength erosion that can be more or less relevant depending on the distortional-to-local critical buckling moment ratio (L-D interaction nature) and the level of applied loading. Therefore, the ultimate strength of elastic-plastic beams with these geometries will be eroded by the occurrence of L-D interaction - naturally, the amount of erosion depends on the yield stress value, which must be large enough for the interaction to develop.

(ii) In beam B1, undergoing $\mathrm{Tl}$, the deformation patterns akin to the local and distortional critical buckling modes emerge simultaneously at early loading stages. The L-D interaction effects increase along the equilibrium path, causing significant erosion of the beam strength. Indeed, this beam reached a limit point at an applied loading level less than $20 \%$ above $M_{c r} \approx M_{c r} \approx M_{c r D}$ - this means the strength benefits of increasing the yield stress are capped by the elastic limit point (i.e., using high-strength steels is probably pointless).

(iii) In beam B2, undergoing SDI, the deformation pattern akin to the local critical buckling mode, expected to remain unchanged until the advanced loading stages (note that $R_{D L}=1.60$ ), was altered by $L-D$ interaction effects at an applied loading level much lower than $M_{c r D}$. Indeed, the surprising emergence of deformations akin to the distortional critical buckling mode at $M / M_{c r l}>0.622$ had the net result of rendering the post-buckling behaviors of the beams B1 and B2 qualitatively fairly similar in terms strength erosion. Quantitatively speaking, beam B2 exhibits higher strengths, as attested by the fact that the (elastic) limit point occurs about $40 \%$ above $M_{c r}=M_{c r L}$ (and considerably below $M_{c r D}$ ).

\footnotetext{
${ }^{17}$ However, note the progressive growth of the "flattened" $w_{2}(x)$ central region.
} 
(iv) The surprising beam B2 post-buckling behavior, addressed in the previous item, is probably due to the fact that development of local deformations in the beam compressed flange (recall the beam local buckling is flange-triggered) causes a significant drop in the cross-section "distortional stiffness", thus causing a drastic distortional critical moment reduction.

(v) In beam B3, undergoing SLI, the deformation pattern akin to the distortional critical buckling mode, remains practically unchanged until well above $M_{c r}=M_{c r D}\left(M / M_{c r D}=1.20\right)$, which means that the beam exhibits a typical distortional post-buckling behavior until that applied load level - this is in contrast with what happened with beam B2, for which the L-D interaction effects came into play at $M / M_{c r l}>0.622$. Beyond $M / M_{c r D}=1.20$, local deformation emerged and the corresponding L-D interaction effects caused a sudden stiffness drop, clearly reflected in the equilibrium path slope. However, the local deformation pattern that emerged at $M / M_{c r D}=1.20$ was a bit surprising, since it was not akin to the beam critical local buckling mode, which exhibits six half-waves. Instead, it exhibited seven half-waves. The explanation for this unexpected behavioral feature lies in the fact that $\left(\mathrm{v}_{1}\right)$ the first two local buckling modes have virtually coincident buckling moments and $\left(\mathrm{v}_{2}\right)$ the latter is symmetric (like the single half-wave critical distortional buckling mode), which favors its emergence. Because the amount of L-D interaction developing in beam B3 is much less than in beams B1 and B2, the strength erosion is smaller and, therefore, the equilibrium does not exhibit a limit point prior to $M / M_{C r D}=1.50$ (the highest applied loading level considered), where the equilibrium path positive slope is still substantial.

(vi) The three beams analyzed in this work exhibited flange-triggered local buckling (the most common situation in practice). Conversely, all the columns analyzed previously exhibited web-triggered local buckling (also the most common situation in practice). This distinct local buckling source is, most likely, responsible for the qualitative behavioral differences observed between the beams and columns under L-D interaction, particularly in the SDI case. In order to clarify all the "grey issues" concerning the mechanics of L-D interaction in lipped channel columns and beams, the authors plan to investigate, in the near future, the behavior of columns and beams exhibiting flange-triggered and web-triggered local buckling, respectively.

Finally, one last word to mention that the authors are currently extending the investigation reported in this work to cover beams subjected to non-uniform bending, a loading case much more common than uniform bending and, therefore, of greater practical interest.

\section{Acknowledgments}

The first author gratefully acknowledges the financial support of FCT (Fundação para a Ciência e a Tecnologia Portugal), through the doctoral scholarship SFRH/BD/87746/2012.

\section{References}

Basaglia C. (2010). Non-linear Analysis of Steel Members and Frames Using Generalized Beam Theory, Ph.D. Thesis in Civil Engineering, Technical University of Lisbon, Portugal. (Portuguese)

Bebiano, R., Gonçalves, R., Camotim, D. (2015). "A cross-section analysis procedure to rationalise and automate the performance of GBT-based structural analysis", Thin-Walled Structures, 92(July), 29-47.

Bebiano R, Camotim D., Gonçalves R. (2016). GBTUL 2.0 - a second-generation code for the GBT-based buckling and vibration analysis of thin-walled members, submitted for publication.

Dinis P.B., Young B., Camotim D. (2016). Local-distortional-global interaction in cold-formed steel lipped channel columns: behavior, strength and DSM design, Website Proceedings of SSRC Annual Stability Conference (Orlando, 12-15/4).

Dinis P.B., Camotim D. (2010). Local/distortional mode interaction in cold-formed steel lipped channel beams, Thin-Walled Structures, 48(10-11), 771-785.

Dinis P.B., Camotim D. (2016). Behaviour and DSM design of hat, zed and rack columns experiencing local-distortional-global interaction, Proceedings of $8^{\text {th }}$ International Conference on Steel and Aluminium Structures (ICSAS'2016 - Hong Kong, 5-7/12), B. Young (ed.). (in press) 
Gonçalves R., Camotim D. (2012). Geometrically non-linear generalized beam theory for elastoplastic thin-walled metal members, Thin-Walled Structures, 51(February), 121-129.

Gonçalves, R., Bebiano, R., Camotim, D. (2014). "On the shear deformation modes in the framework of generalized beam theory", Thin-Walled Structures, 84(November), 325-334.

Martins A.D., Camotim D., Dinis P.B., Providência P. (2015a). On the mechanics of local-distortional interaction in lipped channel thin-walled columns, Website Proceedings of SSRC Annual Stability Conference (Nashville, 24-27/3).

Martins A.D., Dinis P.B., Camotim D., Providência P. (2015b). Local-distortional interaction in pin-ended lipped channel beams: behaviour, strength and DSM design, CD-ROM Proceedings of $8^{\text {th }}$ International Conference on Advances in Steel Structures (ICASS 2015 - Lisbon 21-24/7), D. Camotim et al. (eds.), paper 15.

Martins A.D., Camotim D., Dinis P.B. (2016a). Direct strength method approach for cold-formed steel columns failing in localdistortional interactive modes, Proceedings of Wei-Wen Yu International Specialty Conference on Cold-Formed Steel Structures (CCFSS'2016 - Baltimore, 9-10/09). (in press)

Martins A.D., Camotim D., Dinis P.B., (2016b). Mechanics of the local-distortional interaction in fixed-ended lipped channel columns, Proceedings of International Colloquium on Stability and Ductility of Steel Structures (SDSS'2016 - Timisoara, 30/05-01/6). D. Dubina, V. Ungureanu (eds.), Wiley-Ernst \& Sohn (Mem Martins), 345-352.

Martins A.D., Camotim D., Dinis P.B. (2016c). Local-distortional interaction in cold-formed steel beams: behaviour, strength and DSM design, Proceedings of $8^{\text {th }}$ International Conference on Steel and Aluminum Structures (ICSAS'2016 - Hong Kong, 7-9/12), B. Young (ed.). (in press)

Miosga G. (1976). Vorwiegend Längsbeanspruchte Dünnwandige Prismatische Stäbe und Platten mit Endlichen Elastischen Verformungen, Ph.D. Thesis, Technische Hochschule Darmstadt. (German)

Silvestre N. (2005). Generalized Beam Theory: New Formulations, Numerical Implementation and Applications, Ph.D. Thesis in Civil Engineering, Technical University of Lisbon, Portugal. (Portuguese).

Silvestre N. Camotim D. (2003). GBT buckling analysis of pultruded FRP lipped channel members, Computers \& Structures, 81(18-19), 1889-1904.

Silvestre N. Camotim D. (2004). GBT-based analysis of the local-plate/distortional buckling mode interaction in lipped channel columns, Proceedings of $4^{\text {th }}$ International Conference on Coupled Instabilities in Metal Structures (CIMS'04 - Rome, 2729/09), M. Pignataro, J. Rondal, V. Gioncu (eds), Editura Orizonturi Universitare (Timisoara), 449-462.

Silva, NMF. (2013). Behaviour and Strength of Thin-Walled Laminated FRP Composite Structural Elements, Ph.D. Thesis in Civil Engineering, Technical University of Lisbon, Portugal. (Portuguese)

Simulia Inc. (2008), ABAQUS Standard (version 6.7-5) 